\title{
The Ground State of the Three-Dimensional Random-Field Ising Model
}

John Z. Imbrie*

Lyman Laboratory of Physics, Harvard University, Cambridge, MA02138, USA

\begin{abstract}
We prove that the three-dimensional Ising model in a random magnetic field exhibits long-range order at zero temperature and small disorder. Hence the lower critical dimension for this model is two (or less) and not three as has been suggested by some.
\end{abstract}

\section{Introduction}

Consider the Ising model in a random magnetic field. With spins $\sigma_{i}= \pm 1$ for $i$ in a rectangle $\Lambda \subset \mathbb{Z}^{d}$, the Hamiltonian is

$$
H^{+}(\Lambda)=\sum_{\langle i, j\rangle: i \in \Lambda \text { or } j \in \Lambda} \frac{1}{2}\left(1-\sigma_{i} \sigma_{j}\right)-\sum_{i \in \Lambda} \frac{1}{2} h_{i} \sigma_{i} .
$$

Here we take plus boundary conditions, $\sigma_{i}=1$ for $i \notin \Lambda$; $\langle i, j\rangle$ denotes a nearest neighbor pair; and $h_{i}$ is a random magnetic field. We suppose that the $h_{i}$ are independent random variables, each Gaussian with mean zero and variance $\left\langle h_{i}^{2}\right\rangle=\varepsilon^{2}$.

We present a rigorous proof that in dimension $d=3$, this model exhibits longrange order at zero temperature and small disorder $\varepsilon$. Specifically, we prove that with probability $1-\exp \left(-O\left(\varepsilon^{-2}\right)\right.$ ), uniformly in $\Lambda, \sigma_{0}=1$ in the ground state of $H^{+}(\Lambda)$. This resolves the controversy over the value of the lower critical dimension for the random-field Ising model, at least at $T=0$. The lower critical dimension, $d_{l}$, is the dimension above which long-range ferromagnetic order can exist. Our result establishes that $d_{l} \leqq 2$, ruling out $d_{l}=3$, which was argued for by some authors. The behavior of the model in two dimensions remains an open question, although it is generally believed that there is no long-range order at any temperature, including $T=0$. If this is so, then $d_{l}=2$ at zero temperature. In one dimension, Berretti [1] has shown that there is no long-range order at $T=0$.

\footnotetext{
* Junior Fellow, Society of Fellows. Research supported in part by the National Science Foundation under Grant PHY82-03669
} 
Imry and Ma [2] originally argued that $d_{l} \geqq 2$ as follows. In a region of linear dimension $L$, the typical fluctuation of magnetic field energy should be of the order of $L^{d / 2}$, while the energy of a domain wall surrounding the region is of the order of $L^{d-1}$. (A domain wall or contour is defined to be a connected $(d-1)$-dimensional hypersurface separating a region of plus spins from a region of minus spins.) Thus field fluctuations should destroy any long-range order in $d>2$; also in $d=2$ by considering all possible $L$. It seemed reasonable to believe $d_{l}=2$, though as pointed out in [3], other mechanisms might destroy order in higher dimensions.

Other arguments were subsequently put forward for $d_{l}=3$. Chief among these is the correspondence between random systems in $d$ dimensions and the corresponding pure systems in $d-2$ dimensions. Since $d_{l}=1$ is the nonrandom Ising model, we would have $d_{l}=3$ in the random version. The correspondence was derived by Parisi and Sourlas $[4,5]$ using supersymmetry; nonperturbative $[6,7]$ and rigorous [8] versions were subsequently given. These authors considered scalar field theories rather than Ising models. The Parisi-Sourlas correspondence is exact only in the case of unique solutions to the classical equations of motion, as was pointed out in [5]. This excludes the case of most interest for the Ising model at low temperature, since the desired interaction potential is nonconvex. Nevertheless, the correspondence may be of relevance in a disordered phase, where it would be reasonable to assume a convex potential.

Arguments based on the roughness of the interface were put forward for $d_{l}=3$ $[9,10]$ and for $d_{l}=2[11-13]$. For comprehensive reviews of theoretical, numerical, and experimental work on the lower critical dimension of the random-field Ising model, see [3].

Chalker [16] and Fisher et al. [14] give important extensions of the Imry-Ma argument which will be important in our proof. In an approximation in which there are no contours (domain walls) within contours, they proved that the model is ordered for $d>2$. This entails considering all possible contours surrounding the origin, and proving that with large probability, none of them encloses a net field exceeding the surface area. They used coarse-grained contours to take advantage of the fact that many contours enclose essentially the same volume. We will need coarse-grained contours also, for the same reason: There are too many contours to allow for treating each one independently.

One could argue that the assumption of no contours within contours is essentially what one is attempting to prove. But in fact one obtains that contours of a certain size are rare, assuming only that smaller contours are rare and that they can be neglected. The idea that one can work inductively from smaller to larger contours will be an important ingredient in our proof. Recently Krey [18] has argued that contours within contours play a role in shifting $d_{l}$ to 3 , in contrast to our results.

Fröhlich and Imbrie [15] and Berretti [1] have considered the random-field Ising model at large disorder. They prove that in any dimension, if the disorder is large enough, then there is no long-range order. The results of [15] are uniform as $T \rightarrow 0$. Thus the present paper establishes the existence of a zero temperature transition from long-range order to absence of long-range order, as the disorder is increased. 
It is worth remarking that with minor modifications our proof should be valid in any dimension $d>2$. Also, the choice of a Gaussian distribution for the magnetic fields is inessential. Any continuous, symmetric distribution with Gaussian fall-off would be easy to handle, and other distributions could probably be treated with some additional work. We are also optimistic about obtaining a proof valid for small temperatures, not just $T=0$. Such a proof would combine the expansion methods of [15] with the methods of the present paper.

Results. In stating our main results, we use the following notation. We let $\operatorname{Pr}(E)$ denote the probability of the event $E$, with respect to the measure

$$
d \mu(h)=\prod_{i \in \mathbb{Z}^{3}}\left[\left(2 \pi \varepsilon^{2}\right)^{-1 / 2} e^{-h_{i}^{2} / 2 \varepsilon^{2}} d h_{i}\right] .
$$

If $F$ is a function of the magnetic fields, we let $\bar{F}$ denote its expectation with respect to this measure. Let $\sigma^{\min }\left(\Lambda^{+}\right)$denote the spin configuration of minimum energy $H^{+}(\Lambda)$. This is unique, with probability 1 , because any two energies are linearly independent functions of $\left\{h_{i}\right\}_{i \in \Lambda}$, and the probability that they are equal is zero. Let $\Lambda$ denote a rectangular parallelepiped centered at the origin $0 \in \mathbb{Z}^{3}$, and let $\Lambda_{n}$ denote the cube of $(2 n+1)^{3}$ sites centered at the origin. Let $\eta$ be any small constant, say $\eta=1 / 4$. We state two theorems: the main theorem on long-range order, then a theorem on the decay of $d \mu(h)$-correlations between ground state spins. We assume throughout that $\varepsilon$ is small.

Theorem 1.1 (Long-Range Order). There exists a constant $C>0$ such that for any $i \in \mathbb{Z}^{3}$ and any $\Lambda$,

$$
\operatorname{Pr}\left(\sigma_{i}^{\min }\left(\Lambda^{+}\right)=-1\right) \leqq \exp \left(-C / \varepsilon^{2}\right)
$$

Consequently,

$$
\begin{gathered}
\overline{\sigma_{i}^{\min }\left(\Lambda^{+}\right)} \geqq 1-2 \exp \left(-C / \varepsilon^{2}\right), \\
\overline{\sigma_{i}^{\min }\left(\Lambda^{+}\right) \sigma_{j}^{\min }\left(\Lambda^{+}\right)} \geqq 1-4 \exp \left(-C / \varepsilon^{2}\right) .
\end{gathered}
$$
limit

By the FKG inequality, the sequence $\left\{\sigma_{i}^{\min }\left(\Lambda_{n}^{+}\right)\right\}$is decreasing in $n$. Hence the

$$
\lim _{n \rightarrow \infty} \sigma_{i}^{\min }\left(\Lambda_{n}^{+}\right) \equiv \sigma_{i}^{\min }
$$

exists for each $i \in \mathbb{Z}^{3}$, with probability 1 . Furthermore, the bounds (1.3)-(1.5) of Theorem 1.1 hold for $\sigma^{\min }$ as well. Our methods also give us constructive control over the rate of approach to the infinite volume limit. This will be discussed in more detail in Sect. 6.

Theorem 1.2 (Decay of Correlations). There exists a constant $C>0$ such that for any $i, j \in \mathbb{Z}^{3}$,

$$
\left|\overline{\sigma_{i}^{\min } \sigma_{j}^{\min }}-\overline{\sigma_{i}^{\min }} \overline{\sigma_{j}^{\min }}\right| \leqq \exp \left(-C|i-j|^{1-\eta} / \varepsilon^{2}\right) .
$$

In the last theorem we could as well replace $n^{1-\eta}$ with $n \exp \left[-c_{0}(\log \log n)^{2}\right]$, $c_{0}>0, n=|i-j|$. 
Main Ideas. We need to estimate the probability that $\Gamma$, a contour enclosing the origin, appears as an outer contour in the ground state configuration $\sigma^{\min }\left(\Lambda^{+}\right)$. (An outer contour is one that is not surrounded by other contours.) Suppose that $\Gamma$ is an outer contour of the configuration $\sigma^{\min }\left(\Lambda^{+}\right)$. Let $V(\Gamma)$ denote the volume enclosed by $\Gamma$. The leading term in the expansion for $H^{+}(V(\Gamma))$ in the ground state is

$$
|\Gamma|+\sum_{i \in V(\Gamma)} \frac{1}{2} h_{i} .
$$

This is what one would obtain if there were no contours $\gamma$ surrounded by $\Gamma$. Taking (1.8) to be exact for the moment, we see that $\Gamma$ can only occur if (1.8) is less than any comparison energy in $V(\gamma)$. In particular (1.8) must be less than $\sum_{i \in V(\Gamma)}-\frac{1}{2} h_{i}$, the energy $H^{+}(V(\Gamma))$ of the configuration with no contours whatsoever in $V(\Gamma)$, not even $\Gamma$. We find that in the approximation of no contours within contours, $\Gamma$ can occur only if

$$
|\Gamma|+\sum_{i \in V(\Gamma)} h_{i} \leqq 0 .
$$

As in the Imry-Ma argument, the probability that this happens is less than

$$
\exp \left[-\frac{|\Gamma|^{2}}{2 \varepsilon^{2}|V(\Gamma)|}\right] \leqq \exp \left[-\frac{c}{\varepsilon^{2}} \operatorname{diam}(\Gamma)\right] \ll 1
$$

in three dimensions. Even though there are $\exp (c|\Gamma|)$ contours of area $|\Gamma|$, it can be shown [14] that with large probability, (1.9) is not satisfied for any $\Gamma$.

We intend to use a similar strategy, but making no approximations for the ground state energy in $V(\Gamma)$. One exact formula could be derived by adding to (1.8) a term

$$
\sum_{\gamma}\left[H^{-}(V(\gamma))-\sum_{i \in V(\gamma)} \frac{1}{2} h_{i}\right]
$$

where $\gamma$ runs over the outermost of the contours surrounded by $\Gamma$ in the ground state in $V(\Gamma)$. Here $H^{-}(V(\gamma))$ is evaluated at the ground state configuration $\sigma^{\min }\left(V(\gamma)^{-}\right)$, with minus boundary conditions since $\varrho=-1$ between $\Gamma$ and $\gamma$. Unfortunately (1.15) is not useful because of the nonlocal dependence on the $h_{i}$ 's in each term. In order to say whether or not $\gamma$ is in the ground state, one must know the magnetic fields throughout $V(\Gamma)$, even if $\gamma$ is small. Hence the statistical analysis leading to the bound (1.10) cannot be made because of uncontrollable dependence amongst the random variables.

To understand our local expansion, consider the simplest correction to (1.8) arising from the smallest possible contours:

$$
\sum_{\gamma:|\gamma|=6, V(\gamma) \subset V(\Gamma)} r_{\gamma}(-h)
$$

Here

$$
r_{\gamma}(-h)= \begin{cases}0, & \text { if } \gamma \text { is not in the ground state in } B(\gamma) \\ |\gamma|-\sum_{i \in V(\gamma)} h_{i}, & \text { otherwise }\end{cases}
$$


and $B(\gamma)$ is a cube of diameter 8 centered at $\gamma$. The random variable $r_{\gamma}(-h)$ is defined in terms of a local ground state in $B(\gamma)$, hence it depends on the magnetic fields only in a small neighborhood of $\gamma$. We obtain the true ground state in $V(\Gamma)$ through successive local ground states in larger and larger regions. If larger contours $\gamma^{\prime}$ occur in the ground state, then in representing their effect, $r_{\gamma^{\prime}}$, we have to subtract the terms erroneously included in (1.12). This amounts to a comparison of the ground state energy in $V(\gamma)$ with the energy of some other configuration involving smaller contours only - see definitions (3.1)-(3.3). We assume by induction that we have good control over such comparisons, and attempt to prove the required estimate for $\Gamma$ also.

The complete expansion for the ground state energy in $V(\Gamma)$ contains, in addition to the terms in (1.8), a sum of the local random variables $r_{y}(-h)$ for all $\gamma$ inside $\Gamma$. The comparison energy involves a sum of random variables $r_{\gamma}(h)$. Hence the difference energy involves symmetrized variables $r_{\gamma}(h)-r_{\gamma}(-h)$. Locality and symmetry permits us to estimate these contributions in a manner analogous to the field terms.

In order to avoid uncontrollable sums of $r_{\gamma}$ 's, we have to work with aggregate variables incorporating the effect of all contours of specified diameter, area, and volume that lie within a fixed cube $B$. Then in order to estimate the aggregate variables, we use coarse-grained contours as in [14]. Difficulties arise in arranging for the aggregate variables not to feel the contour $\Gamma$ which is supposed to enclose all contributing contours.

In the next section we define some generalized contours and introduce locally favored contours. We also prove some lemmas on the geometrical relationship between these contours, and on their relation to the true ground state. In the third section we introduce the expansion for the ground state energy, and prove that it can be rewritten in terms of aggregate variables. The fourth section is devoted to the inductive estimates for these variables. In Sect. 5 we prove some entropy estimates that were assumed in the previous section. In the final section, we prove Theorems 1.1 and 1.2, using the results of Sects. 2-5.

\section{Contours and Local Ground States}

In this section we establish some basic definitions for use in the remainder of the paper. We introduce generalized contours which need not be connected in the usual sense. The generalized notion of connectedness is tailored to the requirement of our induction. We also introduce the concept of a favored contour, or a contour present in a local ground state configuration. Favored contours are central to the construction as we approximate the true ground state through local ground states in successively larger regions.

Throughout Sects. 2-5 we fix a large rectangle $\Lambda \subset \mathbb{Z}^{3}$ and restrict to configurations with $\sigma=1$ in $\Lambda^{c}$ (plus boundary conditions) or with $\sigma=-1$ in $\Lambda^{c}$ (minus boundary conditions).

The distance between sites $i, j \in \mathbb{Z}^{3}$ is given by

$$
|i-j|=\max _{\mu}\left|i_{\mu}-j_{\mu}\right| .
$$




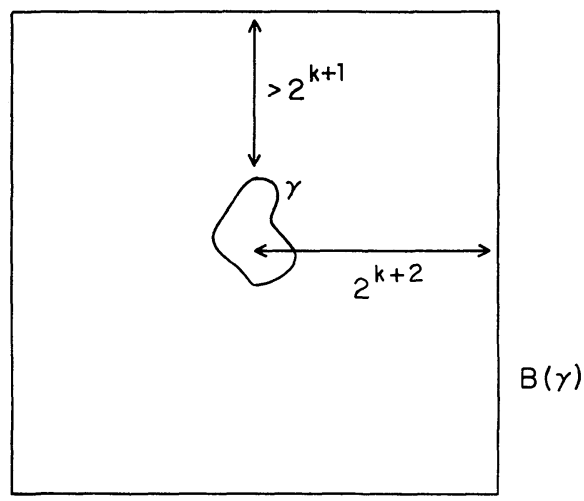

Fig. 1. The box that the contour $\gamma$ belongs to
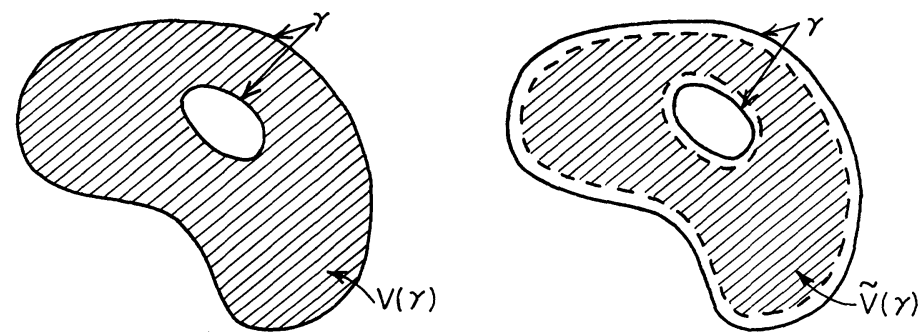

Fig. 2. The volume enclosed by $\gamma$, and the volume $\tilde{V}(\Gamma)$

Consider a subset $V \subset \mathbb{Z}^{3}$, and consider spin configurations $\sigma$ in $V$ with $\sigma=1$ in $(V \cap A)^{c}$. The contours of the configuration in $V$ are the connected components of the set of all plaquettes of the dual lattice separating a nearest neighbor pair of sites $\langle i, j\rangle$ with $\sigma_{i} \sigma_{j}=-1$.

The components of $V$ may have several boundary components. However we will never have to consider configurations with contours surrounding a component of $V^{c}$.

Let $\gamma$ be any union of contours. Then we define $k(\gamma)$ by the inequalities

$$
2^{k(\gamma)} \leqq \operatorname{diam}(\gamma)<2^{k(\gamma)+1}, \quad k(\gamma) \in \mathbb{Z} .
$$

We associate to each contour $\gamma$ a cube $B(\gamma)$ of diameter $2^{k+3}$ containing $\gamma$. The center of $B(\gamma)$ is chosen to be a fixed point near $\gamma$. We take the point of $2^{k} \mathbb{Z}^{3}$ maximizing $\operatorname{dist}(\gamma, \partial B(\gamma))$, and if there are several then we choose the first one (say in a lexicographic ordering of $\mathbb{Z}^{3}$ ). We have that $\operatorname{dist}(\gamma, \partial B(\gamma))>2^{k+1}$ (see Fig. 1). We label such boxes by the site $x \in 2^{k} \mathbb{Z}^{3}$ at the center and by the scale of $\gamma$, for example $B(\gamma)=B_{k, x}$ for $k(\gamma)=k$. We write $\gamma \rightarrow B_{k, x}$ if $B(\gamma)=B_{k, x}$, and say $\gamma$ belongs to $B_{k, x}$.

If $\Gamma$ is any union of disjoint contours, we define $V(\Gamma)$, the volume enclosed by $\Gamma$, to be the collection of sites with $\sigma_{i}=-1$ in an associated spin configuration. The associated spin configuration is defined in the obvious way by putting $\sigma=1$ at $\infty$ and changing the sign of $\sigma$ across each component of $\Gamma$. We use $|\cdot|$ to denote the cardinality of a set, thus $|\Gamma|$ is the area of $\Gamma$ and $|V(\Gamma)|$ is the number of sites enclosed by $\Gamma$ (see Fig. 2). 
We will also need a slightly smaller volume,

$$
\tilde{V}(\Gamma)=\{i \in V(\Gamma) \text { : all nearest neighbors of } i \text { are in } V(\Gamma)\} \text {. }
$$

The outer contours in a region $V$ are the contours $\Gamma$ such that $V(\Gamma) \not \subset V\left(\Gamma^{\prime}\right)$ for any other contour $\Gamma^{\prime}$ in $V$.

So far all contours have been connected. We now join to each outer contour some of the contours it encloses. Fix $\Gamma_{0}$, an outer contour in $V$. Consider the outer contours in $\tilde{V}\left(\Gamma_{0}\right)$; denote them by $\gamma_{\alpha}, \alpha=1,2, \ldots$. We say that $\gamma_{\alpha}$ is connected to $\gamma_{\beta}$ if $\operatorname{dist}\left(B\left(\gamma_{\alpha}\right), B\left(\gamma_{\beta}\right)\right) \leqq 2^{\min \left\{k\left(\gamma_{\alpha}\right), k\left(\gamma_{\beta}\right)\right\}+3}$. We say that $\gamma_{\alpha}$ is connected to $\Gamma_{0}$ if

$$
\operatorname{dist}\left(B\left(\gamma_{\alpha}\right), \Gamma_{0}\right) \leqq 2^{k\left(\gamma_{\alpha}\right)+3} \text { or if }\left|\gamma_{\alpha}\right| \geqq 2^{2\left(N\left(\Gamma_{0}\right)-N_{0}\right)},
$$

where we define

$$
N(\Gamma)=2+\left[\frac{2}{3} \log _{2} \frac{|V(\Gamma)|}{|\Gamma|}\right],
$$

and where $N_{0}$ is an integer constant to be determined later. Here $[x]$ denotes the largest integer less than or equal to $x$, for $x>0,[x] \equiv 0$ otherwise. We define $\Gamma_{1}$ to be the union of $\Gamma_{0}$ and all $\gamma_{\alpha}$ connected directly or indirectly to $\Gamma_{0}$ via the above relations. We repeat the process for $\Gamma_{1}$, adding all $\gamma_{\alpha}$ connected directly or indirectly to $\Gamma_{1}$ using the above relations with $\Gamma_{0}$ replaced by $\Gamma_{1}$. [There can be some new outer contours added because $N\left(\Gamma_{1}\right)$ can be less than $N\left(\Gamma_{0}\right)$.] This defines $\Gamma_{2}$, and we continue in this fashion to define $\Gamma_{3}, \Gamma_{4}$, etc. until $N\left(\Gamma_{j}\right)$ stabilizes. The result is $\Gamma\left(\Gamma_{0}\right)$, and is called the external contour associated with the outer contour $\Gamma_{0}$.

We apply this process to each outer contour in $V$. Due to the holes punched in $V\left(\Gamma_{0}\right)$, there may be some contours $\Gamma$ left with $V(\Gamma) \subset V_{1}=V \bigcup_{\alpha} V\left(\Gamma\left(\Gamma_{0, \alpha}\right)\right)$. (Here $\left\{\Gamma_{0, \alpha}\right\}$ are the outer contours in $V$.) We repeat the above constructions with $V$ replaced by $V_{1}$. We obtain more external contours $\Gamma\left(\Gamma_{0}\right)$ and a residual volume $V_{2}$. Eventually the process stops and all of the contours $\gamma$ in $V$ are either part of an external contour or else $V(\gamma) \subset \tilde{V}(\Gamma)$ for some external contour $\Gamma$. In the latter case they are called internal contours (see Fig. 3).

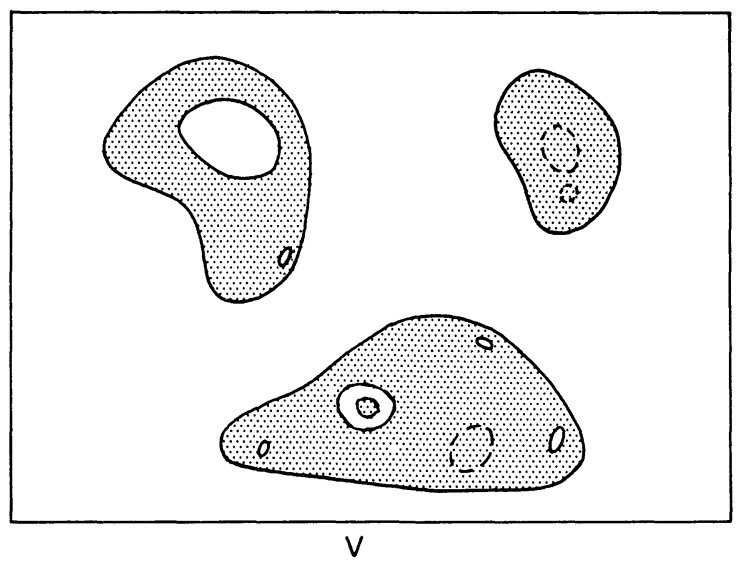

Fig. 3. External and internal contours in $V$. Shaded regions denote $V(\gamma)$ for external contours $\gamma$. Dashed contours are internal contours 
We remark that no external contour $\Gamma$ has internal contours in $V(\Gamma)$ surrounding components of $V(\Gamma)^{c}$. Thus $V(\Gamma)$ satisfies the condition we assumed for $V$.

If $\Gamma_{0}$ belongs to $B_{k, x}$ then we say that the external contour $\Gamma\left(\Gamma_{0}\right)$ belongs to $B_{k, x}$ and we write $\Gamma\left(\Gamma_{0}\right) \rightarrow B_{k, x}$.

In all these constructions we have been assuming $\sigma=1$ in $(V \cap \Lambda)^{c}$; they can of course be repeated for the case $\sigma=-1$ in $(V \cap \Lambda)^{c}$. The choice of boundary condition will always be understood or explicitly stated.

The ground state configuration in a region $V$ with plus boundary conditions is the configuration minimizing

$$
H^{+}(V)=\sum_{\langle i j\rangle: i \in V \text { or } j \in V} \frac{1}{2}\left(1-\sigma_{i} \sigma_{j}\right)-\sum_{i \in V} \frac{1}{2} h_{i} \sigma_{i}
$$

under the constraint $\sigma=1$ in $(V \cap \Lambda)^{c}$. If there is more than one minimum we choose arbitrarily one minimizing the number of sites with $\sigma_{i}=-1$ (the choice can depend only on $V$ and on $h \uparrow V$ ).

An external contour $\Gamma$ is said to be favored in $V^{+}$if it is an external contour of the ground state configuration in $V$ with plus boundary conditions. We have analogous definitions when $V$ has minus boundary conditions.

An external contour $\Gamma$ is said to be favored $(+)$ if it is favored in $B(\Gamma)^{+}$. It is favored (-) if it is favored in $B(\Gamma)^{-}$. The notion of favored depends only on $\Gamma$ and on $h\lceil B(\Gamma)$. Thus it is a local definition depending only a local ground state. Our statistical analysis depends on a reduction to such local notions. Locality makes the corresponding random variables almost independent.

An external contour $\Gamma$ is said to be maximal $\left(\subseteq V^{+}\right)$if it is favored $(+)$, if $V(\Gamma) \subseteq V$, and if no other favored (+) external contour $\Gamma^{\prime}$ satisfies $V(\Gamma) \subseteq V\left(\Gamma^{\prime}\right) \subseteq V$. We say $\Gamma$ is maximal $\left(\varsubsetneqq V^{+}\right)$if it is favored $(+)$, if $V(\Gamma) \varsubsetneqq V$ and if no other favored $(+)$ external contour $\Gamma^{\prime}$ satisfies $V(\Gamma) \varsubsetneqq V\left(\Gamma^{\prime}\right) \varsubsetneqq V$. Such a $\Gamma$ has $V(\Gamma)$ contained in, but not equal to, $V$. Similarly we have external contours that are maximal $\left(\subseteq V^{-}\right)$ or maximal $\left(\varsubsetneqq V^{-}\right)$. Note that these maximality notions are defined in terms of all conceivable favored $(+)$ external contours, not just those in any particular configuration.

We conclude this section with two lemmas on the structure of favored and maximal external contours (see also Fig. 4).
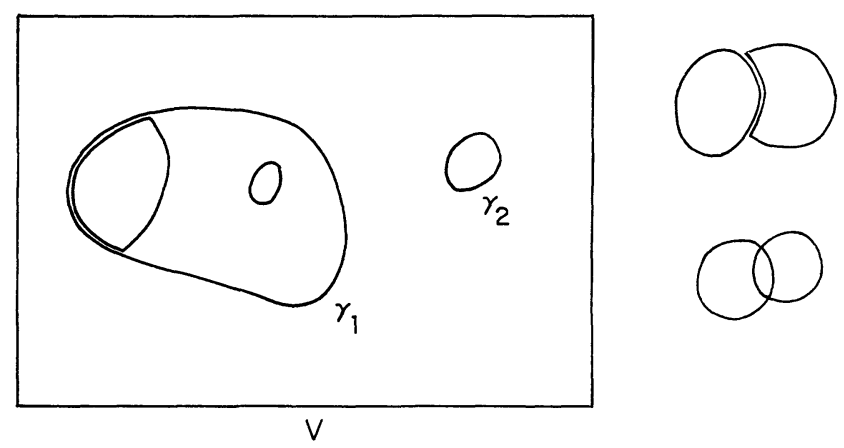

Fig. 4. A possible collection of favored (+)external contours in $V$, with $\gamma_{1}, \gamma_{2}$ maximal $\left(\cong V^{+}\right)$. The pairs on the right cannot depict favored $(+)$ external contours; they are not nested 
Lemma 2.1. The favored $(+)$ external contours are nested, in the sense that any pair of favored $(+)$ external contours $\left(\gamma_{1}, \gamma_{2}\right)$ satisfies one of the following conditions:

(i) $\gamma_{1} \cap \gamma_{2}=\emptyset$ and $V\left(\gamma_{1}\right) \cap V\left(\gamma_{2}\right)=\emptyset$,

(ii) $V\left(\gamma_{1}\right) \subset V\left(\gamma_{2}\right)$ or $V\left(\gamma_{2}\right) \subset V\left(\gamma_{1}\right)$.

Lemma 2.2. Let $V=\Lambda$ or $V=\tilde{V}(\Gamma)$ for $\Gamma$ any favored $(-)$ external contour. The external contours favored in $V^{+}$are the external contours that are maximal $\left(\cong V^{+}\right)$.

Proof of Lemma 2.1. We may as well assume that $k\left(\gamma_{2}\right) \geqq k\left(\gamma_{1}\right)$. Suppose that these contours are not nested. Then $\operatorname{dist}\left(\gamma_{1}, \gamma_{2}\right)=0$, and since

$$
\operatorname{dist}\left(\gamma_{2}, B\left(\gamma_{2}\right)^{c}\right)>2^{k\left(\gamma_{2}\right)+1} \geqq 2^{k\left(\gamma_{1}\right)+1}>\operatorname{diam}\left(\gamma_{1}\right),
$$

we have that $V\left(\gamma_{1}\right) \subset B\left(\gamma_{2}\right)$. In order to arrive at a contradiction, we prove that $\gamma_{2}$ is not in fact an external contour of the ground state configurations in $B\left(\gamma_{2}\right)^{+}$. Let us represent a configuration in $B\left(\gamma_{2}\right)^{+}$by specifying the subset $S=\left\{i \in B\left(\gamma_{2}\right), \sigma_{i}=-1\right\}$. Denote the ground state configuration in $B\left(\gamma_{2}\right)^{+}$by $S_{\min }$ ( $\gamma_{2}$ is supposed to be an external contour of this configuration). Let $S\left(\gamma_{1}\right)$ be the set of all sites $i \in V\left(\gamma_{1}\right)$ with $\sigma_{i}=-1$ in the ground state configuration in $B\left(\gamma_{1}\right)^{+}$. We claim that the configuration $S_{\min } \cup S\left(\gamma_{1}\right)$ has lower energy than the configuration $S_{\min }$. Supposing this to be true, we note that any $i \in V\left(\gamma_{1}\right)$ with a nearest neighbor $j \in V\left(\gamma_{1}\right)^{c}$ must be in $S\left(\gamma_{1}\right)$. If $\gamma_{1}, \gamma_{2}$ are not nested, then at least one such site is in $V\left(\gamma_{2}\right)^{c}$ with a nearest neighbor in $V\left(\gamma_{2}\right)$ (see Fig. 5). This means that the corresponding plaquette in $\gamma_{2}$ would not be part of the contours of $S_{\min } \cup S\left(\gamma_{1}\right)$. Hence $\gamma_{2}$ could not have been an external contour of the ground state in $B\left(\gamma_{2}\right)$, and we have the desired contradiction.

It remains for us to show that $S_{\min } \cup S\left(\gamma_{1}\right)$ has lower energy than $S_{\text {min. }}$. We need to label the portions of contour involved in this comparison. We divide $\partial S_{\min } \mid \partial S\left(\gamma_{1}\right)$ into two sections: $A$ which is the part in $S\left(\gamma_{1}\right)^{c}$, and $C$ which is the part in $S\left(\gamma_{1}\right)$ - see Fig. 6. Similarly we divide $\partial S\left(\gamma_{1}\right) \backslash \partial S_{\min }$ into two parts: $B$ which is the part in $S_{\min }$, and $D$ which is the part in $S_{\min }^{\mathrm{c}}$. Finally we divide $\partial S_{\min } \cap \partial S\left(\gamma_{1}\right)$ into two parts: $E$ which is the part in $S_{\min } \cup S\left(\gamma_{2}\right)$ and $F$ which is the rest. [We say that a plaquette is in $W$ if both of the sites separated by the plaquette are in $W$. We have

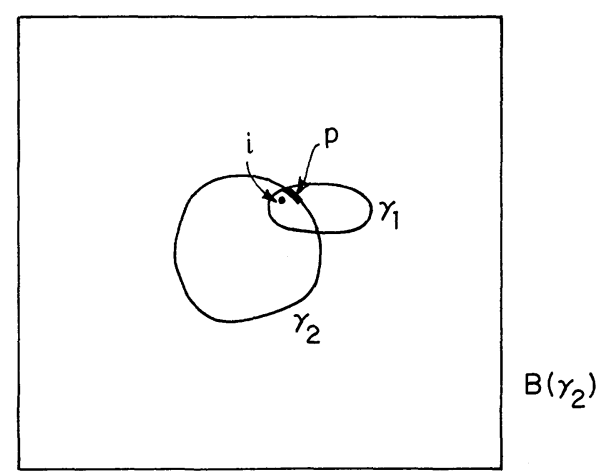

Fig. 5. The site $i$ is in $V\left(\gamma_{2}\right) \cap V\left(\gamma_{1}\right)$ and is adjacent to $V\left(\gamma_{2}\right)^{c}$ and to $V\left(\gamma_{1}\right)^{c}$. It shows that plaquette $p$ could not have been part of $\gamma_{2}$ 


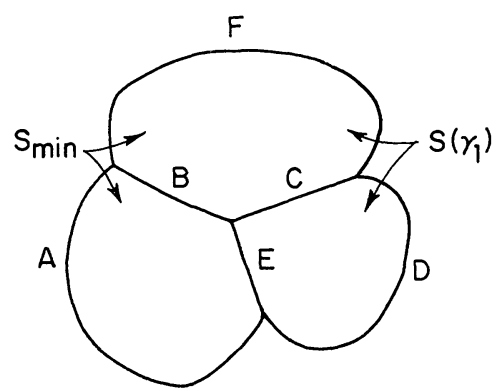

Fig. 6. The sections of contours bounding $S_{\min }$ and $S\left(\gamma_{1}\right)$

that $A \cup C=\partial S_{\min } \backslash \partial S\left(\gamma_{1}\right)$ because any plaquette in $\partial S_{\min }$ which is neither in $S\left(\gamma_{1}\right)$ nor in $S\left(\gamma_{1}\right)^{c}$ must be in $\partial S\left(\gamma_{1}\right)$. Similarly we have $B \cup D=\partial S\left(\gamma_{1}\right) \backslash \partial S_{\text {min }}$.]

We define the energy of the configuration $S$ (normalized to zero for $S=\emptyset$ ) to be

$$
\mathscr{E}(S)=\sum_{i \in S} h_{i}+|\partial S|
$$

We claim that

$$
\mathscr{E}\left(S_{\min } \cup S\left(\gamma_{1}\right)\right)-\mathscr{E}\left(S_{\min }\right)=\mathscr{E}\left(S\left(\gamma_{1}\right)\right)-\mathscr{E}\left(S\left(\gamma_{1}\right) \cap S_{\min }\right)-2|E|
$$

This is easily verified from the equalities

$$
\begin{aligned}
\mathscr{E}\left(S_{\text {min }} \cup S\left(\gamma_{1}\right)\right) & =\sum_{i \in S_{\min } \cup S\left(\gamma_{1}\right)} h_{i}+|A|+|F|+|D|, \\
\mathscr{E}\left(S_{\text {min }}\right) & =\sum_{i \in S_{\min }} h_{i}+|A|+|F|+|C|+|E|, \\
\mathscr{E}\left(S\left(\gamma_{1}\right)\right) & =\sum_{i \in S\left(\gamma_{1}\right)} h_{i}+|B|+|F|+|D|+|E|, \\
\mathscr{E}\left(S\left(\gamma_{1}\right) \cap S_{\text {min }}\right) & =\sum_{i \in S\left(\gamma_{1}\right) \cap S_{\text {min }}} h_{i}+|B|+|F|+|C| .
\end{aligned}
$$

Recall that $S\left(\gamma_{1}\right)$ is the collection of minus sites in $V\left(\gamma_{1}\right)$ in the ground state configuration in $B\left(\gamma_{1}\right)^{+}$. It must also be the collection of minus sites in the ground state configuration in $S\left(\gamma_{1}\right)^{+}$, since any preferred configuration could be extended to all of $B\left(\gamma_{1}\right)$, achieving the same gain in energy or the same reduction in the number of minus sites. Therefore,

$$
\mathscr{E}\left(S\left(\gamma_{1}\right)\right) \leqq \mathscr{E}\left(S\left(\gamma_{1}\right) \cap S_{\min }\right)
$$

$S\left(\gamma_{1}\right)$ being the configuration of lowest energy in $S\left(\gamma_{1}\right)$. As argued above, $S\left(\gamma_{1}\right) \not \subset S_{\min }$ (non-nestedness of $\gamma_{1}, \gamma_{2}$ ) so that these two configurations are distinct. Therefore we must have strict inequality, because equality would mean that $S\left(\gamma_{1}\right) \cap S_{\min }$ would have been selected as ground state configuration due to its smaller number of minus sites.

Combining this information with (2.7), we obtain

$$
\mathscr{E}\left(S_{\min } \cup S\left(\gamma_{1}\right)\right)<\mathscr{E}\left(S_{\min }\right),
$$

which completes the proof of the lemma. 
Proof of Lemma 2.2. The proof is based on the same type of argument as was used in the proof of Lemma 2.1. Namely, if $S_{2}$ is the ground state configuration in $V_{2}^{+}$, and $S_{1} \subset V_{2}$ is the ground state configuration in $V_{1}^{+}$, then $S_{1} \subset S_{2}$. This statement can be proven in the same manner that we proved that $\mathscr{E}\left(S_{\min } \cup S\left(\gamma_{1}\right)\right)<\mathscr{E}\left(S_{\min }\right)$ above.

We remark that any external contour $\gamma$ favored in $V^{+}$satisfies $(B(\gamma) \cap \Lambda) \subset V$. This is clear if $V=\Lambda$. If $V=\widetilde{V}(\Gamma)$ for some favored (-) contour $\Gamma$ then $\operatorname{dist}(B(\gamma), \Gamma)>2^{k+3}$ (otherwise part of $\gamma$ would have been incorporated into $\Gamma$ ). Hence $B(\gamma) \subset V$ in the second case. We remark also that all contours satisfy $V(\Gamma) \subset \Lambda$, hence in both cases $V \cong \Lambda$.

Let $\gamma$ be an external contour favored in $V^{+}$, and let us prove that it is favored $(+)$, i.e. favored in $(B(\gamma) \cap \Lambda)^{+}$. If $S(V)$ is the ground state configuration in $V^{+}$and $S(\gamma)$ is the ground state configuration in $(B(\gamma) \cap \Lambda)^{+}$, then $S(\gamma) \subset S(V)$. Furthermore, any component of $S(V)$ that is contained in $B(\gamma) \cap A$ is contained in $S(\gamma)$. In particular, the two configurations agree in $V\left(\gamma_{0}\right)$ (where $\gamma_{0}$ is the outer contour for $\gamma$ ) and at sites bordering $V\left(\gamma_{0}\right)$. Thus $S(\gamma)$ is obtained by removing from $S(V)$ some components of minus sites that do not intersect or border on $V\left(\gamma_{0}\right)$. Such an operation cannot change the fact that $\gamma$ is an external contour. Hence $\gamma$ is favored $(+)$.

Next we show that if $\gamma$ is maximal $\left(\subseteq V^{+}\right)$then it is favored in $V^{+}$. As before we have that $S(\gamma) \subset S(V)$. Suppose there is a site $i \in S(V), i \in V(\gamma)^{c}, i$ adjacent to $V(\gamma)$. Consider the component $C$ of $S(V)$ containing $i$. $C$ contains also a site in $V(\gamma)$ adjacent to $i$, because sites in $V(\gamma)$ bordering on $V(\gamma)^{c}$ are in $S(\gamma)$. We have that $C \subset V\left(\gamma^{\prime}\right)$ for some external contour $\gamma^{\prime}$ favored in $V^{+}$. By our analysis above, $\gamma^{\prime}$ is favored $(+)$. By Lemma 2.1, $\gamma$ and $\gamma^{\prime}$ are nested. However, since $V(\gamma) \cap V(\gamma) \neq \emptyset$, $V\left(\gamma^{\prime}\right) \cap V(\gamma)^{c} \neq \emptyset$, this is only possible if $V(\gamma) \varsubsetneqq V\left(\gamma^{\prime}\right)$. See Fig. 7. This contradicts the fact that $\gamma$ is maximal $\left(\cong V^{+}\right)$. Hence no such site $i$ exists.

We have learned that $\sigma_{i}=1$ for $i \in V(\gamma)^{c}$ adjacent to $V(\gamma)$. Hence any component of $S(V)$ intersecting $V\left(\gamma_{0}\right)$ is contained in $S(\gamma)$. [Here $\gamma_{0}$ is the outer contour for $\gamma$.] Thus the two configurations agree in $V\left(\gamma_{0}\right)$ and at sites adjacent to $V\left(\gamma_{0}\right)$. The only way that $\gamma$ could fail to be an external contour of the ground state configuration in $V^{+}$is if $\gamma_{0}$ were an internal contour. In other words, we would have $V(\gamma) \varsubsetneqq V\left(\gamma^{\prime \prime}\right)$ for some external contour $\gamma^{\prime \prime}$ favored in $V^{+}$. But as before, this

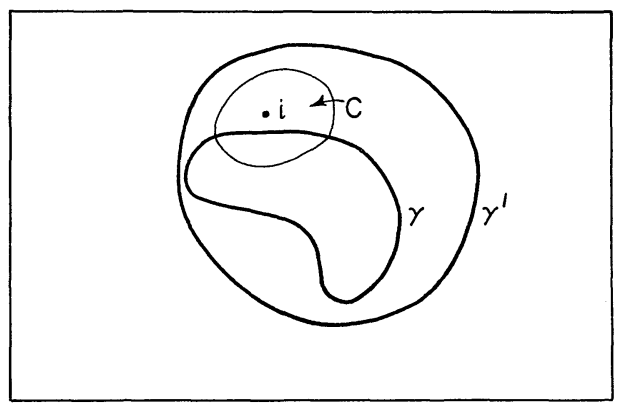

Fig. 7. The existence of a component $C$ of minus spins in the ground state configuration in $V^{+}$ demonstrates that $\gamma$ is not maximal $\left(\subseteq V^{+}\right)$ 
would imply that $\gamma^{\prime \prime}$ is favored $(+)$, contradicting maximality of $\gamma$. Thus $\gamma$ is actually favored in $V^{+}$.

To complete the proof of the lemma, we have to show that if $\gamma$ is favored in $V^{+}$, then $\gamma$ is maximal $\left(\subseteq V^{+}\right)$. We have already shown that $\gamma$ is favored $(+)$. If $\gamma$ were not maximal $\left(\subseteq V^{+}\right)$, then we would have $V(\gamma) \subseteq V\left(\gamma^{\prime}\right)$ with $\gamma^{\prime}$ maximal $\left(\subseteq V^{+}\right)$. We have just shown that $\gamma^{\prime}$ is favored in $V^{+}$. But then $\gamma$ could not be an external contour of the ground state configuration in $V^{+}$- external contours have disjoint enclosed volumes. Hence $\gamma$ must actually be maximal $\left(\subseteq V^{+}\right)$.

\section{An Expansion for the Ground State Energy}

This section is devoted to a definition of some random variables that depend on the magnetic fields in a local fashion, and to a proof that the ground state energy can be expanded in terms of these variables.

Define for $V \subseteq \Lambda$

$$
H_{\min }^{+}(V)=\inf _{\sigma \mid V} H^{+}(V)+\sum_{i \in V} \frac{1}{2} h_{i} .
$$

The second term normalizes this ground state energy to zero in the case that the minimizing configuration has no contours. Next we define

$$
E^{+}(V)=\sum_{\gamma \text { maximal }\left(\varsubsetneqq V^{+}\right)} H_{\min }^{+}(V(\gamma))
$$

and

$$
r_{\gamma}= \begin{cases}0, & \text { if } \gamma \text { is not favored }(+), \\ H_{\min }^{+}(V(\gamma))-E^{+}(V(\gamma)), & \text { otherwise. }\end{cases}
$$

We have the following interpretation of $E^{+}(V)$.

Proposition 3.1. $E^{+}(V)-\sum_{i \in V} \frac{1}{2} h_{i}$ is the energy $H^{+}(V)$ of a spin configuration in $V$ with plus boundary conditions, obtained as follows. In each $V(\gamma), \gamma$ a maximal $\left(\subseteq V^{+}\right)$ external contour, we put $\sigma=\sigma^{\min }\left(V(\gamma)^{+}\right)$, where $\sigma^{\min }\left(V(\gamma)^{+}\right)$is the ground state configuration in $V(\gamma)$ with plus boundary conditions. Elsewhere we put $\sigma=1$. As a consequence, we have

$$
r_{\gamma} \leqq 0
$$

Proof. We remark that the spin configuration of the proposition is well defined because volumes enclosed by maximal $\left(\varsubsetneqq V^{+}\right)$external contours do not intersect (Lemma 2.1). Furthermore, the maximal $\left(\varsubsetneqq V^{+}\right)$external contours do not intersect (Lemma 2.1). Hence $\mathrm{H}^{+}(V)$ splits into a sum of $\mathrm{H}^{+}(V(\gamma))$ 's and the energy of the spin configuration above is equal to

$$
\begin{aligned}
& \sum_{\gamma \text { maximal }\left(\varsubsetneqq V^{+}\right)} \inf _{\sigma \mid V(\gamma)} H^{+}(V(\gamma))-\sum_{\substack{i \in V, i \notin V(\gamma) \\
\text { for } \gamma \operatorname{maximal}\left(\varsubsetneqq V^{+}\right)}} \frac{1}{2} h_{i} \\
= & \sum_{\gamma \operatorname{maximal}\left(\varsubsetneqq V^{+}\right)} H_{\min }^{+}(V)-\sum_{i \in V} \frac{1}{2} h_{i} \\
= & E^{+}(V)-\sum_{i \in V} \frac{1}{2} h_{i} .
\end{aligned}
$$


We have used the definitions (3.1), (3.2).

The inequality (3.4) follows because $H_{\min }^{+}(V)-\sum_{i \in V} \frac{1}{2} h_{i}$ is the minimum energy; the energy of the configuration in the proposition must be at least as large. Hence $H_{\min }^{+}(V) \leqq E^{+}(V)$.

Proposition 3.1 allows us to interpret $r_{\gamma}$ as the amount that the energy can be lowered in $V(\gamma)$ by extending the ensemble of allowed configurations to include $\gamma$. Next we derive telescoping expansions for $H_{\min }^{+}(V), E^{+}(V)$ in terms of the $r_{\gamma}$ 's.

Proposition 3.2. The following expansions hold for $V=\Lambda$ or $V=V(\Gamma)$ with $\Gamma$ favored $(+)$ :

$$
\begin{gathered}
H_{\min }^{+}(V)=\sum_{\gamma: V(\gamma) \leqq V} r_{\gamma}, \\
E^{+}(V)=\sum_{\gamma: V(\gamma) \varsubsetneqq V} r_{\gamma} .
\end{gathered}
$$

Proof. If $V=V(\Gamma)$ with $\Gamma$ favored (+), then these two statements are equivalent, by (3.3), since the two expansions differ by $r_{\Gamma}=H_{\min }^{+}(V(\Gamma))-E^{+}(V(\Gamma))$. If $\partial V$ is not favored $(+)$, then $r_{\partial V}=0$ and the expansions agree. But if $\partial V$ is not favored $(+)$, then $V=\Lambda$ and maximal $\left(\varsubsetneqq V^{+}\right)$is equivalent to maximal $\left(\subseteq V^{+}\right)$. Hence by Lemma 2.2 the configuration of Proposition 3.1 is actually the ground state configuration in $V^{+}$. [Here we use the fact that if $\gamma$ is favored $(+)$, then $\gamma$ is favored in $V(\gamma)^{+}$and so $\sigma^{\min }\left(V(\gamma)^{+}\right)=\sigma^{\min }\left(V^{+}\right)\left\lceil V(\gamma)\right.$.] Hence in this case $H_{\min }^{+}(V)$ $=E^{+}(V)$, and (3.6), (3.7) are equivalent in all cases. Thus it is sufficient to prove (3.7), assuming (3.6) for smaller $V$ 's. Note that if $V$ is so small that there is no favored $(+) \gamma$ with $V(\gamma) \varsubsetneqq V$, then (3.2) and (3.7) agree since there are no external contours contributing to either sum.

By (3.2) and (3.6) we have

$$
E^{+}(V)=\sum_{\gamma \text { maximal }}\left(\varsubsetneqq V^{+}\right) \sum_{\gamma^{\prime}: V\left(\gamma^{\prime}\right) \subseteq V(\gamma)} r_{\gamma^{\prime}}
$$

Comparing this with (3.7), we note that every favored $(+) \gamma^{\prime}$ with $V\left(\gamma^{\prime}\right) \varsubsetneqq V$ satisfies $V\left(\gamma^{\prime}\right) \subseteq V(\gamma)$ for some $\gamma$ which is maximal $\left(\varsubsetneqq V^{+}\right)$. By Lemma 2.1, the external contours which are maximal $\left(\varsubsetneqq V^{+}\right)$have nonintersecting $V(\gamma)$ 's. Therefore the maximal $\left(\varsubsetneqq V^{+}\right)$external contour $\gamma$ surrounding each favored $(+) \gamma^{\prime}$ is unique. If $\gamma^{\prime}$ is not favored $(+)$, then $r_{\gamma^{\prime}}=0$ and it does not contribute. This proves (3.7) and the proposition.

We now derive a formula relating $r_{\gamma}$ to $r_{\gamma^{\prime}}$ 's with $V\left(\gamma^{\prime}\right) \subseteq V(\gamma)$. This formula will be the basis for inductive estimates on the $r_{\gamma}$ 's. Let us consider the case that $\gamma$ is favored $(+)$, since otherwise $r_{\gamma}=0$.

The ground state configuration in $V(\gamma)^{+}$must have $\gamma$ as external contour [any improvement in $V(\gamma)^{+}$could be transferred to $B(\gamma)^{+}$, since $\gamma$ is favored in $\left.B(\gamma)^{+}\right]$. To see what happens inside $V(\gamma)$, look at the slightly smaller volume $\tilde{V}(\gamma)$. Clearly $\sigma_{j}=-1$ in $V(\gamma) \backslash \tilde{V}(\gamma)$; otherwise $\gamma$ would not be the external contour of the ground state in $V(\gamma)^{+}$. Thus $\widetilde{V}(\gamma)$ has minus boundary conditions. By the minus boundary condition version of Lemma 2.2, the ground state configuration in $\tilde{V}(\gamma)^{-}$has for its external contours the ones that are maximal $\left(\subseteq \tilde{V}(\gamma)^{-}\right)$. 
We need to perform all the constructions of this section for minus boundary conditions also. It is necessary to change $\sum_{i \in V} \frac{1}{2} h_{i}$ to $\sum_{i \in V}-\frac{1}{2} h_{i}$ in (3.1) and elsewhere. By symmetry, changing the boundary condition is equivalent to reversing the sign of the $h_{i}$ 's. Thus we have instead of the definition (3.3) an equality

$$
r_{\gamma}(-h)= \begin{cases}0, & \text { if } \gamma \text { is not favored }(-), \\ H_{\min }^{-}(V(\gamma))-E^{-}(V(\gamma)), & \text { otherwise }\end{cases}
$$

Here $h$ is understood to represent all the $h_{i}$ 's on which $r_{\gamma}$ depends.

Having described the ground state in $V(\gamma)$, we have the following formula for the ground state energy:

$$
\begin{aligned}
\inf _{\sigma \mid V(\gamma)} H^{+}(V(\gamma)) & =|\gamma|+\sum_{\substack{i \in V(\gamma), i \notin V\left(\gamma^{\prime}\right) \\
\text { for } \gamma^{\prime} \operatorname{maximal}\left(\underline{\varrho}(\gamma)^{-}\right)}} \frac{1}{2} h_{i}+\sum_{\gamma^{\prime} \operatorname{maximal}\left(\leqq \tilde{V}(\gamma)^{-}\right)} \inf _{\sigma \mid V\left(\gamma^{\prime}\right)} H^{-}\left(V\left(\gamma^{\prime}\right)\right) \\
& =|\gamma|+\sum_{i \in V(\gamma)} \frac{1}{2} h_{i}+\sum_{\gamma^{\prime} \operatorname{maximal}\left(\cong \tilde{V}(\gamma)^{-}\right)} H_{\min }^{-}\left(V\left(\gamma^{\prime}\right)\right) .
\end{aligned}
$$

We have used Lemma 2.1 to conclude that the maximal $\left(\subseteq \widetilde{V}(\gamma)^{-}\right)$external contours are nonintersecting and have nonintersecting enclosed volumes. From (3.10) we obtain

$$
H_{\text {min }}^{+}(V(\gamma))=|\gamma|+\sum_{i \in V(\gamma)} h_{i}+\sum_{\gamma^{\prime} \operatorname{maximal}\left(\leqq \tilde{V}(\gamma)^{-}\right)} H_{\min }^{-}\left(V\left(\gamma^{\prime}\right)\right) .
$$

Applying Proposition 3.2 and (3.3), we obtain

$$
r_{\gamma}(h)=\left\{\begin{array}{l}
0, \quad \text { if } \gamma \text { is not favored }(+), \\
|\gamma|+\sum_{i \in V(\gamma)} h_{i}+\sum_{\gamma^{\prime}: V\left(\gamma^{\prime}\right) \subseteq \tilde{V}(\gamma)} r_{\gamma^{\prime}}(-h)-\sum_{\gamma^{\prime}: V\left(\gamma^{\prime}\right) \subsetneq V(\gamma)} r_{\gamma^{\prime}}(h), \\
\text { if } \gamma \text { is favored }(+) .
\end{array}\right.
$$

Notice that we have almost a sum of symmetrized random variables $r_{\gamma^{\prime}}(h)-r_{\gamma^{\prime}}(-h)$. Since these variables have mean zero, like the $h_{i}$ 's it should be unlikely for them to exceed $|\gamma|$ in magnitude (the Imry-Ma argument). As this is clearly a necessary condition for $r_{\gamma}<0$, we see that $r_{\gamma}$ is usually zero. This is essentially how our argument goes. However, we need to work with aggregates of the $r_{\gamma}$ 's to obtain good estimates.

Define

$$
\begin{gathered}
r_{k a, w, v, x}= \begin{cases}\sum_{\gamma \rightarrow B_{k}, x:|\gamma| \in[a, 2 a),} r_{\gamma}, & \text { if the number of such favored }(+) \gamma \text { is in }[v, 2 v), \\
|V(\gamma)| \in[w, 2 w) & \text { otherwise, }\end{cases} \\
\qquad \delta r_{k, a, w, v, x}(h)=r_{k, a, w, v, x}(h)-r_{k, a, w, v, x}(-h) .
\end{gathered}
$$

Here $a=2^{m}, w=2^{l}, v=2^{p}$ for some $m, l, p \in \mathbb{Z}$. Now we define

$$
c_{\gamma}(h)= \begin{cases}0, & \text { if } \gamma \text { is not favored }(+), \\ |\gamma|+\sum_{i \in V(\gamma)} h_{i}-\sum_{k, a, w, v, x}^{*} \delta r_{k, a, w, v, x}(h), & \text { if } \gamma \text { is favored }(+) .\end{cases}
$$


The star indicates the following restrictions: $w<2^{3\left(N(\gamma)-N_{0}\right)}, k \leqq k(\gamma)$, and $\left|B_{k, x} \backslash V(\gamma)\right|<w$. We define, in analogy with (3.13)

$$
c_{k, a, w, v, x}(h)=\left\{\begin{array}{l}
\sum_{\gamma \rightarrow B_{k, x}:|\gamma| \in[a, 2 a),|V(\gamma)| \in[w, 2 w)} c_{\gamma}(h), \\
\quad \text { if the number of such favored }(+) \gamma \text { is in }[v, 2 v), \\
0, \quad \text { otherwise. }
\end{array}\right.
$$

The following proposition shows that $c_{\gamma}(h)$ is a useful approximation to $r_{\gamma}(h)$.

Proposition 3.3. If $\gamma$ is favored $(+)$, then

$$
r_{\gamma}(h)=c_{\gamma}(h)-\sum_{\gamma^{\prime}: V\left(\gamma^{\prime}\right) \varsubsetneqq V(\gamma)}^{* c} r_{\gamma^{\prime}}(h) .
$$

Here $* c$ indicates the complementary restrictions:

$$
\left|V\left(\gamma^{\prime}\right)\right| \geqq 2^{3\left(N(\gamma)-N_{0}\right)} \quad \text { or } \quad\left|B\left(\gamma^{\prime}\right) \backslash V(\gamma)\right| \geqq w\left(\gamma^{\prime}\right) .
$$

Here $w\left(\gamma^{\prime}\right)$ is defined by $\left|V\left(\gamma^{\prime}\right)\right| \in\left[w\left(\gamma^{\prime}\right), 2 w\left(\gamma^{\prime}\right)\right)$. As a consequence, we have

$$
\begin{gathered}
c_{\gamma}(h) \leqq r_{\gamma}(h) \leqq 0, \\
c_{k, a, w, v, x}(h) \leqq r_{k, a, w, v, x}(h) \leqq 0 .
\end{gathered}
$$

Proof. Consider the terms $r_{\gamma^{\prime}}(h)$ that should appear in (3.12): all $\gamma^{\prime}$ with $V\left(\gamma^{\prime}\right) \subsetneq V(\gamma)$. Clearly exactly one of the following conditions are satisfied:

$$
\left|V\left(\gamma^{\prime}\right)\right| \in[w, 2 w) \quad \text { with } \quad w<2^{3\left(N(\gamma)-N_{0}\right)} ; \quad\left|V\left(\gamma^{\prime}\right)\right| \geqq 2^{3\left(N(\gamma)-N_{0}\right)} .
$$

By Lemma 2.1 if $\gamma^{\prime}$ is favored $(+)$, then either $V\left(\gamma^{\prime}\right) \subset V(\gamma)$ or $V\left(\gamma^{\prime}\right) \subset V(\gamma)^{c}$. Since no external contours $\gamma^{\prime}$ with $V\left(\gamma^{\prime}\right) \subset V(\gamma)^{c}$ and $\left|V\left(\gamma^{\prime}\right)\right| \in[w, 2 w)$ can belong to $B_{k, x}$ 's contributing to (3.15), all $\gamma^{\prime}$ contributing to (3.15) satisfy $V\left(\gamma^{\prime}\right) \subseteq V(\gamma)$. Furthermore, if $V\left(\gamma^{\prime}\right)=V(\gamma)$, then $\gamma^{\prime}=\gamma$ so that

$$
w \geqq w^{2 / 3} \geqq c|V(\gamma)|^{2 / 3}|\gamma|^{-2}|\gamma|^{2} \geqq c\left(\frac{|V(\gamma)|}{|\gamma|}\right)^{2} \geqq 2^{3\left(N(\gamma)-N_{0}\right)} .
$$

[We have used the elementary inequality $|\gamma| \geqq 6|V(\gamma)|^{2 / 3}$.] Thus $\gamma^{\prime}=\gamma$ does not occur in (3.15); all $\gamma^{\prime}$ contributing to (3.15) satisfy $V\left(\gamma^{\prime}\right) \varsubsetneqq V(\gamma)$. With this condition satisfied, we have $k\left(\gamma^{\prime}\right) \leqq k(\gamma)$ automatically, and we need only notice that the conditions on $B\left(\gamma^{\prime}\right)$ in (3.15) and (3.17) are complementary. Thus we have shown that each $\gamma^{\prime}$ contributing to (3.12) contributes to exactly one of the sums in (3.15), (3.17).

Next we consider the terms $r_{\gamma^{\prime}}(-h)$ that should appear in (3.12): all $\gamma^{\prime}$ with $V\left(\gamma^{\prime}\right) \subseteq \tilde{V}(\gamma)$. All nonzero terms should appear in (3.15) as there are no others in (3.17). Any outer contour $\gamma_{0}$ in the ground state in $\tilde{V}(\gamma)^{-}$with $\operatorname{dist}\left(B\left(\gamma_{0}\right), \gamma\right) \leqq 2^{k\left(\gamma_{0}\right)+3}$ would have been joined with $\gamma$, by our construction of external contours. [We are assuming that $\gamma$ is favored $(+)$; this implies that it is favored in $V(\gamma)^{+}$.] Hence we can assume there are no such outer contours in the ground state in $\tilde{V}(\gamma)^{-}$. By Lemma 2.2 there are no such maximal $\left(\subseteq \tilde{V}(\gamma)^{-}\right)$ external contours. The existence of any favored $(-)$ external contour $\gamma_{0}$ with $\operatorname{dist}\left(B\left(\gamma_{0}\right), \gamma\right) \leqq 2^{k\left(\gamma_{0}\right)+3}$ would imply the existence of a maximal $\left(\subseteq \tilde{V}(\gamma)^{-}\right)$contour even closer to $\gamma$. Hence there are no such favored $(-)$ external contours. Thus the 
limitation on $B_{k, x}$ in (3.15) does not remove desired $r_{\gamma^{\prime}}(-h)$ terms; all $\gamma^{\prime}$ contributing to (3.12) belong to a $B_{k, x} \subseteq V(\gamma)$.

In an analogous fashion we show that the limitation $w<2^{3\left(N(\gamma)-N_{0}\right)}$ in (3.15) does not remove desired $r_{\gamma^{\prime}}(-h)$ terms. Any outer contour $\gamma_{0}$ in the ground state in $\tilde{V}(\gamma)^{-}$with $\left|\gamma_{0}\right| \geqq 2^{2\left(N(\gamma)-N_{0}\right)}$ would have been joined with $\gamma$. Hence there are no such outer contours in the ground state in $\tilde{V}(\gamma)^{-}$. If $\gamma^{\prime}$ is favored $(-), V\left(\gamma^{\prime}\right) \cong \widetilde{V}(\gamma)$, $\left|V\left(\gamma^{\prime}\right)\right| \in[w, 2 w)$, then

$$
w \leqq\left|V\left(\gamma^{\prime}\right)\right| \leqq\left|V\left(\gamma_{0}\right)\right| \leqq\left|\gamma_{0}\right|^{3 / 2}<2^{3\left(N(\gamma)-N_{0}\right)},
$$

where $\gamma_{0}$ is the outer contour of the maximal $\left(\subseteq \tilde{V}(\gamma)^{-}\right)$external contour enclosing $\gamma^{\prime}$. [We have again used Lemma 2.2 to deduce that $\gamma_{0}$ is in the ground state in $\left.\tilde{V}(\gamma)^{-}.\right]$Thus all $\gamma^{\prime}$ contributing to (3.12) are included in (3.15).

Lastly, we need to show that every $r_{\gamma^{\prime}}(-h)$ contributing to (3.15) is present in (3.12) also, i.e. we must show that $V\left(\gamma^{\prime}\right) \subseteq \tilde{V}(\gamma)$. We need the following lemma, similar to ones used to prove Lemmas 2.1 and 2.2.

Lemma 3.4. Let $S_{2}$ be the set of plus spins in the ground state configuration in $V_{2}^{+}$, and let $S_{1} \subset V_{2}$ be the set of plus spins in the ground state configuration in $S_{1}^{-}$. Then $S_{1} \subset S_{2}$.

Proof. We could prove the lemma directly, but it is simpler to reduce to the situation considered in the proof of Lemma 2.1. First change all signs, so that $h \rightarrow-h, S_{2}$ is the set of minus spins in the ground state in $V_{2}^{-}$, and $S_{1}$ is the set of minus spins in the ground state in $S_{1}^{+}$. Next define

$$
\bar{V}_{2}=V_{2} \cup\left\{i: i \text { has a nearest neighbor in } V_{2}\right\} \text {, }
$$

and put $h_{i}=-\infty$ in $\bar{V}_{2} \backslash V_{2}$. This enforces $\sigma_{i}=-1$ in $\bar{V}_{2} \backslash V_{2}$, so that $S_{2} \cup\left(\bar{V}_{2} \backslash V_{2}\right)$ is the set of minus spins in the ground state in $\bar{V}^{+}$. (Actually it is a ground state maximizing, rather than minimizing, the number of minus spins.) The argument in the proof of Lemma 2.2 shows that the configuration $S_{1} \cup S_{2} \cup\left(\bar{V}_{2} \backslash V_{2}\right)$ has an energy less than the energy of $S_{2} \cup\left(\bar{V}_{2} \backslash V_{2}\right)$, unless $S_{1} \subset S_{2}$. As $S_{2} \cup\left(\bar{V}_{2} \backslash V_{2}\right)$ is the ground state, this cannot happen, and we have $S_{1} \subset S_{2}$.

In the situation at hand, we take $V_{2}=B(\gamma) \cap \Lambda$ and $S_{1}$ to be the set of plus spins in the ground state in $V\left(\gamma^{\prime}\right)^{-}$. This of course implies that $S_{1}$ is the set of plus spins in the ground state in $S_{1}^{-}$. Since $V\left(\gamma^{\prime}\right) \not \subset V(\gamma)^{c}$ and $k\left(\gamma^{\prime}\right) \leqq k(\gamma)$, we have that $V\left(\gamma^{\prime}\right) \subset B(\gamma)$. Furthermore, $V\left(\gamma^{\prime}\right)$ is always contained in $\Lambda$, so we have $S_{1} \subset V_{2}$ and the lemma
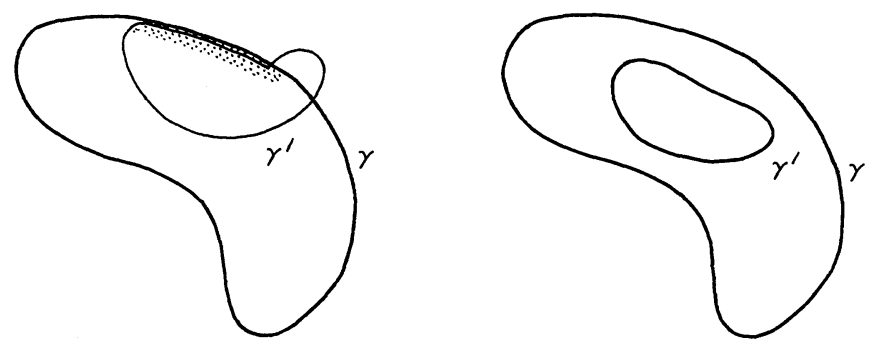

Fig. 8. Sites in the shaded region are in $S_{1}$ and in $S_{2}^{c}$. Hence $\gamma^{\prime}, \gamma$ are as pictured on the right 
applies. Suppose that $V\left(\gamma^{\prime}\right) \not \subset \widetilde{V}(\gamma)$. We have also $V(\gamma) \not \subset V(\gamma)^{c}$, and in addition $V(\gamma) \not \subset V(\gamma)$ because otherwise we would have $w\left(\gamma^{\prime}\right) \geqq 2^{3\left(N(\gamma)-N_{0}\right)}$ as in (3.19). Therefore there is at least one site in $V(\gamma) \cap V\left(\gamma^{\prime}\right)$ that is adjacent to $V(\gamma)^{c}$ and to $V(\gamma)^{c}$ (see Fig. 8). Being adjacent to $V\left(\gamma^{\prime}\right)^{c}$ means it is in $S_{1}$, and being adjacent to $V(\gamma)^{c}$ means it is in $S_{2}^{c}$. This contradicts Lemma 3.4, which says that $S_{1} \subset S_{2}$. Hence $V\left(\gamma^{\prime}\right) \cong \tilde{V}(\gamma)$.

This completes the proof of (3.17). The inequalities (3.18) follow immediately, because $r_{\gamma^{\prime}}(h) \leqq 0$.

\section{Probability Estimates for the Induction Step}

In this section we formulate an induction hypothesis on the distribution of the random variables $c_{k, a, w, v, x}(h)$. This is used to estimate the distribution of sums of random variables $\delta r_{k, a, w, v, x}(h)$. Finally we verify the induction hypothesis, assuming some entropy estimates from Sect. 5.

Proposition 4.1. Let

$$
N(a, w) \equiv 2+\left[\frac{2}{3} \log _{2} \frac{w}{a}\right]<\bar{N} .
$$

There exists a constant $c_{0}>0$ such that for any $R \geqq 0$,

$$
\operatorname{Pr}\left(c_{k, a, w, v, x}(h)<-R\right) \leqq \exp \left\{-\frac{(v a+R)^{2}}{\varepsilon^{2} v w} \exp \left[-c_{0}(\log N(a, w))^{2}\right]\right\} .
$$

We will work using an induction on $\bar{N}$. Note that by (3.18), the estimate (4.1) holds for $r_{k, a, w, v, x}(h)$ and for $\delta r_{k, a, w, v, x}(h)$; we have

$$
c_{k, a, w, v, x}(h) \leqq r_{k, a, w, v, x}(h) \leqq \delta r_{k, a, w, v, x}(h) .
$$

Furthermore, since $\delta r_{k, a, w, v, x}(h)$ is symmetric, we have the same estimate for the probability that $\delta r_{k, a, w, v, x}(h)>R$.

Let $x_{i} \in 2^{k} \mathbb{Z}^{3}, i=1, \ldots, n$ be such that $\left|x_{i}-x_{i^{\prime}}\right| \geqq 2^{k+3}$ for all $i \neq i^{\prime}$. Then since $r_{\gamma}$ depends only on $h\left\lceil B(\gamma)\right.$, and since $B_{k, x_{i}} \cap B_{k, x_{i}}=\emptyset$, the $\delta r_{k, a, w, v, x_{i}}(h)$ 's are independent random variables. In order to estimate the probability distribution of $\sum_{i=1}^{n} \delta r_{k, a, w, v, x_{i}}$, we work with the moment generating functional for $\delta r_{k, a, w, v, x_{i}}$. Define

$$
f_{i}(s)=\int e^{s y} \lambda_{i}(y) d y,
$$

where $\lambda_{i}(y)$ is the probability density for $\delta r_{k, a, w, v, x_{i}}$, that is,

$$
\int_{R_{1}^{+}}^{R_{\overline{2}}^{-}} \lambda_{i}(y) d y=\operatorname{Pr}\left(\delta r_{k, a, w, v, x_{i}} \in\left(R_{1}, R_{2}\right)\right) .
$$

The above-mentioned estimates on $\delta r_{k, a, w, v, x_{i}}$ imply that

$$
\begin{aligned}
\operatorname{Pr}\left(\delta r_{k, a, w, v, x_{i}} \neq 0\right) & \leqq 2 \exp \left\{-\frac{v a^{2}}{\varepsilon^{2} w} \exp \left[-c_{0}(\log N(a, w))^{2}\right]\right\} \\
& \leqq \exp \left(-\frac{\bar{c}}{\varepsilon^{2}} 2^{k / 2}\right) \ll 1 .
\end{aligned}
$$


We use $\bar{c}$ to denote constants that may depend on $c_{0}$. All other constants $N_{0}, c, c_{1}, \ldots$ can be chosen independently of $c_{0}$. The second inequality follows from

$$
\begin{gathered}
\frac{a^{2}}{w} \geqq \frac{c|\gamma|^{2}}{|V(\gamma)|} \geqq c \operatorname{diam}(\gamma) \geqq c 2^{k}, \\
N(a, w) \leqq \log _{2} \frac{w}{a}+c \leqq \log _{2} \operatorname{diam}(\gamma)+c \leqq k+c .
\end{gathered}
$$

We prove (4.6) by noting that the maximum $|V(\gamma)|$, given $|\gamma|$ and $\operatorname{diam}(\gamma)$, is achieved at a cylinder-like shape with $|V(\gamma)| \approx L^{2} \operatorname{diam}(\gamma), L$ being approximately the radius of the cylinder. We have $|\gamma| \geqq c L \operatorname{diam}(\gamma)$ so that $|\gamma|^{2} /|V(\gamma)| \geqq c \operatorname{diam}(\gamma)$. For (4.7) we need only note that $|V(\gamma)| \leqq|\gamma| \operatorname{diam}(\gamma)$.

From (4.5) we see that $\lambda_{i}(y)$ is dominated by a delta function at $y=0$, hence the need to be careful about endpoints in (4.4).

For convenience we define

$$
B=B(v, a, w)=\varepsilon^{2} v w \exp \left[c_{0}(\log N(a, w))^{2}\right] .
$$

Lemma 4.2. Let $s$ be real. For $|s| \leqq 2 v a / B$,

$$
\left|f_{i}(s)\right| \leqq 1+e^{-(v a)^{2} / 2 B},
$$

and for any s,

$$
\left|f_{i}(s)\right| \leqq \exp \left(s^{2} B / 4\right) .
$$

Proof. By symmetry, we can take $s \geqq 0$. If $s \leqq 2 v a / B$, then

$$
\begin{aligned}
\left|f_{i}(s)-1\right| & =\int_{-\infty}^{\infty}\left(e^{s y}-1\right) \lambda_{i}(y) d y \\
& \leqq 2 \int_{0^{+}}^{\infty}\left(e^{2 v a y / B}-1\right) \lambda_{i}(y) d y \\
& \leqq \frac{4 v a}{B} \int_{0}^{\infty} e^{2 v a y / B} \Lambda_{i}(y) d y,
\end{aligned}
$$

by integration by parts, where

$$
\Lambda_{i}(y)=\int_{y^{+}}^{\infty} \lambda_{i}\left(y^{\prime}\right) d y^{\prime}=\operatorname{Pr}\left(\delta r_{k, a, w, v, x_{i}}>y\right) .
$$

Hence we have

$$
\begin{aligned}
\left|f_{i}(s)-1\right| & \leqq \frac{4 v a}{B} \int_{0}^{\infty} e^{2 v a y / B} e^{-(y+v a)^{2} / B} d y \\
& \leqq e^{-(v a)^{2} / B} \frac{4 v a}{B} \int_{0}^{\infty} e^{-y^{2} / B} d y \\
& \leqq e^{-(v a)^{2} / B} \frac{c v a}{B^{1 / 2}} \leqq e^{-(v a)^{2} / 2 B}
\end{aligned}
$$

The last inequality holds because by (4.5), $a^{2} / B \gg 1$. This proves (4.9). 
The bound (4.12) extends immediately to complex $s,|s| \leqq 2 v a / B$. Hence Cauchy's theorem implies that

$$
\left|f_{i}^{\prime \prime}(s)\right| \leqq c\left(\frac{B}{v a}\right)^{2} e^{-(v a)^{2} / 2 B}, \quad|s| \leqq v a / B .
$$

Furthermore we have $f_{i}(0)=1$, and by symmetry $f_{i}^{\prime}(0)=0$. Hence for $s \leqq v a / B$,

$$
f_{i}(s) \leqq 1+c s^{2}\left(\frac{B}{v a}\right)^{2} e^{-(v a)^{2} / 2 B} \leqq e^{s^{2} B / 4},
$$

provided

$$
c \frac{B}{(v a)^{2}} e^{-(v a)^{2} / 2 B} \leqq 1 .
$$

But this is true because $a^{2} / B \gg 1$. This proves (4.10) for $|s| \leqq v a / B$.

Next consider $s>v a / B$. We have

$$
\begin{aligned}
f_{i}(s) & \leqq 1+2 s \int_{0^{+}}^{\infty} e^{s y} \Lambda_{i}(y) d y \\
& \leqq 1+2 s \int_{0}^{\infty} e^{s y} e^{-(y+v a)^{2} / B} d y \\
& \leqq 1+2 s \int_{0}^{\infty} e^{s^{2} B / 4} e^{-s v a} e^{-(y-s B / 2+v a)^{2} / B} d y \\
& \leqq 1+c s B^{1 / 2} e^{-s v a} e^{s^{2} B / 4} \leqq 1+e^{-(v a)^{2} / 2 B} e^{s^{2} B / 4} .
\end{aligned}
$$

We have used $a \geqq 1$, and

$$
B \leqq v 2^{3 k} \exp \left[c_{0}(\log N(a, w))^{2}\right] \leqq \bar{c} v 2^{(3+\eta) k} \leqq\left((v a)^{2} / B\right)^{8},
$$

which follows from (4.7), (4.5). (Here $\eta$ is a fixed small positive number, say $\eta=1 / 4$.) Now the right-hand side of (4.15) is bounded by $e^{s^{2} B / 4}$, since

$$
e^{s^{2} B / 4}\left(1-e^{-(v a)^{2} / 2 B}\right) \geqq \exp \left[\frac{(v a)^{2}}{4 B}-2 e^{-(v a)^{2} / 2 B}\right] \geqq 1 .
$$

This completes the proof.

Lemma 4.2 gives us the information we need to estimate the distribution of sums of $\delta r_{k, a, w, v, x}(h)$ 's. The bound (4.9) gives rise to an exponentially decaying distribution, while (4.10) yields a Gaussian falloff which is better for large values of the sum. These two behaviors reflect corresponding behaviors in (4.1): Exponential for small $R$, Gaussian for large $R$. There is a third domain of very rapid, Gaussian falloff for the distributions of sums of $\delta r_{k, a, w, v, x}(h)$ 's for very small values of the sum. This behavior (reflecting the central limit theorem for these random variables) arises from an improvement of the bound in (4.9) to $\exp \left[s^{2} \exp \left(-\bar{c} 2^{k / 2} / \varepsilon^{2}\right)\right]$, see (4.14). However, we prove in the following lemma only what will be needed for the induction step. 
Lemma 4.3. If $x_{i} \in 2^{k} \mathbb{Z}^{3},\left|x_{i}-x_{i^{\prime}}\right| \geqq 2^{k+3}$, then for $R \geqq 0$,

$$
\begin{gathered}
\operatorname{Pr}\left(\sum_{i=1}^{n} \delta r_{k, a, w, v, x_{i}}(h)<-R\right) \leqq \exp \left[-R^{2} /(n B)\right] \\
\operatorname{Pr}\left(\sum_{i=1}^{n} \delta r_{k, a, w, v, x_{i}}(h)<-R\right) \leqq \exp \left[n e^{-(v a)^{2} / 2 B}-\frac{2 v a}{B} R\right] .
\end{gathered}
$$

Proof. Let $F(s)$ denote the moment generating functional as in (4.3) for $\lambda_{F}$, the density for $\sum \delta r_{k, a, w, v, x_{i}}(h)$. As these are independent random variables, this density is a convolution of $\lambda_{i}(y)$ 's, so by Lemma 4.2 ,

$$
F(s)=\prod_{i=1}^{n} f_{i}(s) \leqq \begin{cases}\left.\exp n e^{-(v a)^{2} / 2 B}\right], & |s| \leqq 2 v a / B \\ \exp \left(n s^{2} B / 4\right), & \text { any } s .\end{cases}
$$

This yields

$$
\begin{aligned}
\operatorname{Pr}\left(\sum_{i=1}^{n} \delta r_{k, a, w, v, x_{i}}(h)<-R\right) & =\int_{-\infty}^{-R^{-}} \lambda_{F}(y) d y \\
& \leqq e^{-s R} \int_{-\infty}^{-R^{-}} e^{-s y} \lambda_{F}(y) d y \leqq e^{-s R} F(-s) .
\end{aligned}
$$

Putting $s=2 v a / B$ and using the first bound in (4.20), we obtain (4.19). If we take $s=2 R /(n B)$ and use the second bound in (4.20), we obtain (4.18).

Corollary 4.4. There is a constant $c_{1}>0$ such that for arbitrary $\left\{x_{i}\right\}$ and $R \geqq 0$,

$$
\begin{gathered}
\operatorname{Pr}\left(\sum_{i=1}^{n} \delta r_{k, a, w, v, x_{i}}(h)<-R\right) \\
\leqq c_{1} \exp \left[\frac{-R^{2}}{c_{1} \varepsilon^{2} n v w} \exp \left[-c_{0}(\log N(a, w))^{2}\right]\right], \\
\operatorname{Pr}\left(\sum_{i=1}^{n} \delta r_{k, a, w, v, x_{i}}(h)<-R\right) \leqq c_{1} \exp \left[n e^{-\bar{c} 2^{k / 2} / \varepsilon^{2}}-\frac{a}{\bar{c} \varepsilon^{2} w} 2^{-\eta k} R\right] .
\end{gathered}
$$

The same estimates hold for $\operatorname{Pr}\left(\sum \delta r_{k, a, w, v, x_{i}}(h)>R\right)$.

Proof. The sites $\left\{x_{i}\right\}$ can be divided into at most $8^{3}$ subsets, each of which satisfies the condition in Lemma 4.3. In order for the event $\sum_{i=1}^{n} \delta r_{k, a, w, v, x_{i}}(h)<-R$ to hold, at least one subset $S$ must satisfy $\sum_{i \in S} \delta r_{k, a, w, v, x_{i}}(h)<-R / 8^{3}$. Each subset satisfies $|S| \leqq n / 8^{3}$, hence by Lemma 4.3 the probability is bounded as in (4.21), with $c_{1}=8^{3}$. Similarly, (4.22) follows using (4.5) and

$$
\frac{v a}{B}=\frac{v a}{\varepsilon^{2} w} \exp \left[-c_{0}(\log N(a, w))^{2}\right] \geqq \frac{a}{\bar{c} \varepsilon^{2} w} 2^{-\eta k} .
$$

The other estimates hold by symmetry. 
Lemma 4.5. There is a $c_{2}>0$ such that for any $R \geqq 0$,

$$
\operatorname{Pr}\left(\sum_{i=1}^{n} h_{i}<-R\right) \leqq \exp \left(-\frac{R^{2}}{c_{2} n \varepsilon^{2}}\right),
$$

and similarly for $\operatorname{Pr}\left(\sum_{i=1}^{n} h_{i}>R\right)$.

Proof. As we have chosen a Gaussian distribution for each $h_{i}$, the sum has probability density $\left(2 \pi n \varepsilon^{2}\right)^{-1 / 2} \exp \left(-y^{2} /\left(2 n \varepsilon^{2}\right)\right)$, and (4.24) follows easily.

Let us now consider a particular random variable $c_{\bar{k}, \bar{a}, \bar{w}, \bar{v}, \bar{x}}(h)$. From (3.16), (3.15) we see that for each $k, a, w, v$ there is a certain subset of $2^{k} \mathbb{Z}^{3} \cap B_{\bar{k}, \bar{x}}$ of sites $x$ with $\delta r_{k, a, w, v, x}$ contributing to $c_{\bar{k}, \bar{a}, \bar{w}, \bar{v}, \bar{x}}$. Denote this subset by $V_{k, w}(\bar{\gamma})$, where $\bar{\gamma}=\left\{\gamma_{i}\right\}$ is the collection of favored $(+)$ external contours $\gamma_{i} \rightarrow B_{k, x}$ with $\left|\gamma_{i}\right| \in[a, 2 a)$, $\left|V\left(\gamma_{i}\right)\right| \in[w, 2 w)$. [These are just the $\gamma$ 's contributing to (3.16).] We have

$$
V_{k, w}(\bar{\gamma})=\bigcup_{\gamma \in \bar{\gamma}} V_{k, w}(\gamma)
$$

where $V_{k, w}(\gamma)$ is the set of all $x$ such that $\left|B_{k, x} \backslash V(\gamma)\right|<w$. Let us define also $V(\bar{\gamma})$ $=\bigcup_{\gamma \in \gamma} V(\gamma)$. Note that $w \leqq 2^{3 k}=\left|B_{k, x}\right| / 8^{3}$, so that an $x$ can satisfy this condition for

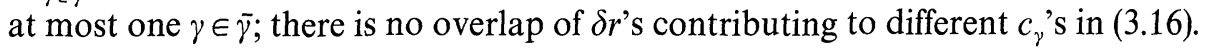

The subset $V_{k, w}(\gamma)$ is a generalization of the notion of coarse-grained contours introduced in [14]. If we put $w=\left|B_{k, x}\right| / 2$, then $V_{k, w}(\gamma)$ would be essentially the same as the volume enclosed by $\gamma$ after coarse-graining as in [14] on the scale $2^{k+3}$. For smaller $w$, more of the structure of $\gamma$ is preserved in $V_{k, w}(\gamma)$. The corresponding increase in entropy will be balanced by improved probability estimates for small $w$.

It is not sufficient to apply Corollary 4.4 to the $V_{k, w}(\bar{\gamma})$ 's introduced above; some additional coarse-graining is needed. For this we use the same procedure as in [14], rescaled to $2^{k} \mathbb{Z}^{3}$. Specifically we introduce

$$
\begin{aligned}
V_{k, w, j}(\bar{\gamma}) & =\bigcup_{\gamma \in \bar{\gamma}} V_{k, w, j}(\gamma), \\
V_{k, w, j}(\gamma) & =\bigcup_{y \in W_{k, w, j}(\gamma)} c_{k, j}(y), \\
W_{k, w, j}(\gamma) & =\left\{y \in 2^{k+j} \mathbb{Z}^{3}:\left|c_{k, j}(y) \cap V_{k, w}(\gamma)\right|>\frac{1}{2} 2^{3 j}\right\} .
\end{aligned}
$$

Here $c_{k, j}(y)$ is the cube consisting of all sites $x \in 2^{k} \mathbb{Z}^{3}$ with

$$
x_{\mu} \in\left[y_{\mu}-\frac{1}{2} 2^{k+j}, y_{\mu}+\frac{1}{2} 2^{k+j}\right), \quad \mu=1,2,3 .
$$

To handle the field terms, we introduce

$$
\begin{aligned}
V_{j}(\bar{\gamma}) & =\bigcup_{\gamma \in \bar{\gamma}} V_{j}(\gamma), \\
V_{j}(\gamma) & =\bigcup_{y \in W_{j}(\gamma)} c_{0, j}(y), \\
W_{j}(\gamma) & =\left\{y \in 2^{j} \mathbb{Z}^{3}:\left|c_{0, j}(y) \cap V(\gamma)\right|>\frac{1}{2} 2^{3 j}\right\} .
\end{aligned}
$$

Note that $V_{k, w, 0}(\bar{\gamma})=V_{k, w}(\bar{\gamma}), V_{0}(\bar{\gamma})=V(\bar{\gamma})$. Also the sets $V_{k, w, j}(\gamma), \quad \gamma \in \bar{\gamma}$ are nonoverlapping, as are the sets $V_{j}(\gamma), \gamma \in \bar{\gamma}$. 
These definitions lead to the following expansion for $c_{\bar{k}, \bar{a}, \bar{w}, \bar{v}, \bar{x}}(h)$. The collection of favored $(+)$ external contours $\bar{\gamma}$ is determined by $\bar{k}, \bar{a}, \bar{w}, \bar{v}, \bar{x}$, and $h$. If $|\bar{\gamma}| \notin[\bar{v}, 2 \bar{v})$, then $c_{\bar{k}, \bar{a}, \bar{w}, \bar{v}, \bar{x}}(h)=0$. Otherwise, we have

$$
\begin{aligned}
c_{\bar{k}, \bar{a}, \bar{w}, \bar{v}, \bar{x}}(h)= & \sum_{\gamma \in \bar{\gamma}}|\gamma|+\sum_{j=1}^{N(\bar{a}, \bar{w})}\left[\sum_{i \in V_{j-1}(\bar{\gamma}) \backslash V_{j}(\bar{\gamma})} h_{i}-\sum_{i \in V_{j}(\bar{\gamma}) \backslash V_{j-1}(\bar{\gamma})} h_{i}\right] \\
& +\sum_{i \in V_{N(\bar{a}, \bar{w})(\bar{\gamma})}} h_{i}+\sum_{k \leqq \bar{k}} \sum_{w<\max _{\gamma \in \bar{\gamma}} 2^{3\left(N(\gamma)-N_{0}\right)}} \\
& \cdot \sum_{a, v}\left\{\sum _ { j = 1 } ^ { N ( \overline { a } , \overline { w } ) - 3 k } \left[\sum_{x \in V_{k, w}, j} \sum_{\left.x \in V_{k, w}\right) \backslash V_{k, w, j-1}(\bar{\gamma})} \delta r_{k, a, w, v, x}(h)\right.\right. \\
& \left.\left.-\sum_{j-1(\bar{\gamma}) \backslash V_{k, w, j}(\bar{\gamma})} \delta r_{k, a, w, v, x}(h)\right]-\sum_{x \in V_{k, w}, N(\bar{a}, \bar{w})-3 k(\bar{\gamma})} \delta r_{k, a, w, v, x}(h)\right\} \\
\equiv & A+\sum_{j=1}^{N(\bar{a}, \bar{w})}\left[H_{j}^{+}-H_{j}^{-}\right]+H_{N}+\sum_{k, w, a, v}\left\{\sum_{j=1}^{N(\bar{a}, \bar{w})-3 k}\left[\Delta_{j}^{+}-\Delta_{j}^{-}\right]-\Delta_{N}\right\} .
\end{aligned}
$$

In the last line we have defined $A, H_{j}^{ \pm}, H_{N}, \Delta_{j}^{ \pm}, \Delta_{N}$ by the corresponding expressions above. If $N(\bar{a}, \bar{w})-3 k<1$, then we define $\Delta_{N}$ to be the whole sum,

$$
\Delta_{N}=\sum_{x \in V_{k, w}(\bar{\gamma})} \delta r_{k, a, w, v, x}(h), \quad \Delta_{j}^{+}=\Delta_{j}^{-}=0 .
$$

The following lemma will be extremely important in controlling the deterioration of our main estimate (Proposition 4.1) as the size of contours increases.

Lemma 4.6. All pairs $(a, w)$ contributing to $c_{\bar{k}, \bar{a}, \bar{w}, \bar{v}, \bar{x}}(h)$ in (4.25) satisfy

$$
N(a, w) \leqq \frac{2}{3} N(\bar{a}, \bar{w}) .
$$

In particular, Proposition 4.1 (the induction hypothesis) can be applied to such $(a, w)$.

Each time an induction step is performed, our estimate (Proposition 4.1) deteriorates; basically the exponent in (4.1) acquires some inverse powers of $k$. The estimate (4.27) helps by making iterations sparse - only $\log _{3 / 2} \log _{2} \frac{w}{a}$ induction steps are used in estimating $c_{k, a, w, v, x}$. Hence the total factor of deterioration, represented by the factor $\exp \left[-c_{0}(\log N(a, w))^{2}\right]$ in (4.1), is no worse than $2^{-\eta k}, \eta$ small. Ultimately the sparsity of iterations was achieved by our construction of external contours, whereby large contours within an outer contour were joined with the outer contour.

Proof of Lemma 4.6. Recalling that $N(a, w) \equiv 2+\left[\frac{2}{3} \log _{2} \frac{w}{a}\right], a \geqq c w^{2 / 3}$, we have

$$
2^{\frac{3}{2} N(a, w)} \leqq c \frac{w}{a} \leqq c w^{1 / 3} \leqq c 2^{N(\bar{a}, \bar{w})-N_{0}} .
$$


The last inequality follows from the condition $w \leqq \max _{\gamma \in \bar{\gamma}} 2^{3\left(N(\gamma)-N_{0}\right)}$ in (4.26); the external contour $\gamma$ has $|\gamma| \in[\bar{a}, 2 \bar{a}),|V(\gamma)| \in[w, 2 w)$. Choosing $N_{0}$ appropriately, we have a bound $2^{N(\bar{a}, \bar{w})}$ on (4.28), which yields (4.27).

Noting that $A \geqq v a$, we can estimate the probability that $c_{\bar{k}, \bar{a}, \bar{w}, \bar{v}, \bar{x}}(h)<-R$ by

$$
\begin{aligned}
\sum_{j=1}^{N(\bar{a}, \bar{w})} & {\left[\operatorname{Pr}\left(\exists \bar{\gamma}: H_{j}^{+}<-\frac{\bar{v} \bar{a}+R}{c j^{2}}\right)+\operatorname{Pr}\left(\exists \bar{\gamma}: H_{j}^{-}>\frac{\bar{v} \bar{a}+R}{c j^{2}}\right)\right] } \\
& +\operatorname{Pr}\left(\exists \bar{\gamma}: H_{N}<-\frac{1}{4}(\bar{v} \bar{a}+R)\right) \\
& +\sum_{k, w, a, v}\left\{\sum _ { j = 1 } ^ { N ( \overline { a } , \overline { w } ) - 3 k } \left[\operatorname{Pr}\left(\exists \bar{\gamma}: \Delta_{j}^{+}<-\frac{\bar{v} \bar{a}+R}{c j^{2} k^{5}}\right)\right.\right. \\
& \left.\left.+\operatorname{Pr}\left(\exists \bar{\gamma}: \Delta_{j}^{-}>\frac{\bar{v} \bar{a}+R}{c j^{2} k^{5}}\right)\right]+\operatorname{Pr}\left(\exists \bar{\gamma}: \Delta_{N}>\frac{\bar{v} \bar{a}+R}{c k^{5}}\right)\right\} .
\end{aligned}
$$

This works because

$$
\sum_{j=1}^{N(\bar{a}, \bar{w})} \frac{2}{c j^{2}}+\frac{1}{4}+\sum_{k, w, a, v}\left\{\sum_{j=1}^{N(\bar{a}, \bar{w})-k} \frac{2}{c j^{2} k^{5}}+\frac{1}{c k^{5}}\right\} \leqq 1,
$$

so that at least one of the above events must occur if the $H, \Delta$ terms in (4.26) are to be less than $-A-R$. Note that there are at most $c k^{3}$ choices of $w, a, v$, given $k$, because $w \leqq 2^{3 k}, a \leqq c 2^{3 k}, v \leqq 2^{2 k}$. This yields (4.30).

In order to estimate (4.16) we need bounds on the volumes of difference regions $V_{j-1}(\bar{\gamma}) \backslash V_{j}(\bar{\gamma})$, etc., and some entropy estimates. The following two propositions will be proven in Sect. 5 .

Proposition 4.7. There is a constant $c_{3}$ such that for any $\bar{\gamma}$ contributing to $c_{\bar{k}, \bar{a}, \bar{w}, \bar{v}, \bar{x}}(h)$,

$$
\begin{gathered}
\left|V_{j-1}(\bar{\gamma}) \backslash V_{j}(\bar{\gamma})\right|, \quad\left|V_{j}(\bar{\gamma}) \backslash V_{j-1}(\bar{\gamma})\right| \leqq c_{3} 2^{j} \bar{v} \bar{a} \\
\left|V_{k, w, j-1}(\bar{\gamma}) \backslash V_{k, w, j}(\bar{\gamma})\right|, \quad\left|V_{k, w, j}(\bar{\gamma}) \backslash V_{k, w, j-1}(\bar{\gamma})\right| \leqq c_{3} 2^{j} w^{-2 / 3} \bar{v} \bar{a} .
\end{gathered}
$$

Proposition 4.8. Let $\bar{\gamma}$ vary over all possible collections of external contours that could contribute to $c_{\bar{k}, \bar{a}, \bar{w}, \bar{v}, \bar{x}}(h)$ in (3.16) for some $h$, that is,

$$
\bar{\gamma}=\left\{\gamma_{i} \rightarrow B_{\bar{k}, \bar{x}}, i=1, \ldots, \mu \text { with } \mu \in[\bar{v}, 2 \bar{v}),\left|\gamma_{i}\right| \in[\bar{a}, 2 \bar{a}),\left|V\left(\gamma_{i}\right)\right| \in[\bar{w}, 2 \bar{w})\right\} .
$$

The number of resulting $V_{j}(\bar{\gamma})^{\prime} s$ is less than $\exp \left(c j 2^{-2 j} \bar{v} \bar{a}\right)$. The number of resulting $V_{k, w, j}(\bar{\gamma})$ 's is less than $\exp \left(c(j+k) 2^{-2 j} w^{-2 / 3} \bar{v} \bar{a}\right)$.

The estimates involving $V_{j}(\bar{\gamma})$ 's are similar to ones proven in [14], the only difference being that we have more complicated contours.

Note that Proposition 4.7 allows us to estimate the "final volumes" $V_{N(\bar{a}, \bar{w})}(\bar{\gamma})$ and $V_{k, w, N(\bar{a}, \bar{w})-3 k}(\bar{\gamma})$. We have

$$
\left|V_{N(\bar{a}, \bar{w})}(\bar{\gamma})\right| \leqq|V(\bar{\gamma})|+\sum_{j=1}^{N(\bar{a}, \bar{w})}\left|V_{j}(\bar{\gamma}) \backslash V_{j-1}(\bar{\gamma})\right| \leqq c v w+c 2^{N(\bar{a}, \bar{w})} \bar{v} \bar{a} \leqq c \bar{v} \bar{w},
$$


because $2^{N(\bar{a}, \bar{w})} \leqq c(\bar{w} / \bar{a})^{2 / 3} \leqq c \bar{w} / \bar{a}$. Similarly,

$$
\begin{aligned}
\mid V_{k, w, N(\bar{a}, \bar{w})-3 k}(\bar{\gamma}) & \leqq\left|V_{k, w}(\bar{\gamma})\right|+\sum_{j=1}^{N(\bar{a}, \bar{w})-3 k}\left|V_{k, w, j}(\bar{\gamma}) \backslash V_{k, w, j-1}(\bar{\gamma})\right| \\
& \leqq c 2^{-3 k} \bar{v} \bar{w}+c 2^{N(\bar{a}, \bar{w})-3 k} w^{-2 / 3} \bar{v} \bar{a} \leqq c 2^{-3 k} \bar{v} \bar{w}
\end{aligned}
$$

Let us use these propositions and Lemma 4.5 to bound the $H$-terms in (4.29) by

$$
\begin{aligned}
& 2 N(\bar{a}, \bar{w}) \sup _{1 \leqq j \leqq N(a, w)} \exp \left[c j 2^{-2 j} \bar{v} \bar{a}-(\bar{v} \bar{a}+R)^{2} /\left(c j^{4} 2^{j} \bar{v} \bar{a} \varepsilon^{2}\right)\right] \\
& +\exp \left[c N(\bar{a}, \bar{w}) 2^{-2 N(\bar{a}, \bar{w})} \bar{v} \bar{a}-(\bar{v} \bar{a}+R)^{2} /\left(c \bar{v} \bar{w} \varepsilon^{2}\right)\right]
\end{aligned}
$$

For each term in (4.35) the second (energy) term in the exponential is much larger than the first (entropy) term. For example, we have

$$
N(\bar{a}, \bar{w}) 2^{-2 N(\bar{a}, \bar{w})} \bar{v} \bar{a} \leqq c 2^{-3 N(\bar{a}, \bar{w}) / 2} \bar{v} \bar{a} \leqq c \bar{v} \bar{a}^{2} / \bar{w} .
$$

The largest term is the last, because

$$
j^{4} 2^{j} \bar{v} \bar{a} \leqq c 2^{3 N(\bar{a}, \bar{w}) / 2} \bar{v} \bar{a} \leqq c \bar{v} \bar{w} .
$$

Hence (4.35) is bounded by $\exp \left[-(\bar{v} \bar{a}+R)^{2} /\left(c \bar{v} \bar{w} \varepsilon^{2}\right)\right]$, using

$$
2 N(\bar{a}, \bar{w})+1 \leqq \exp \left(c 2^{k}\right) \leqq \exp \left(c \bar{v} \bar{a}^{2} / \bar{w}\right) .
$$

Next consider a $\Delta$-term with $j<2^{k / 2}$ in (4.29), estimating it using (4.32) and the geometric mean of (4.21) and (4.22):

$$
\begin{aligned}
\operatorname{Pr}\left(\exists \gamma: \Delta_{j}^{+}<-\frac{\bar{v} \bar{a}+R}{c j^{2} k^{5}}\right) \leqq & c_{1} \exp \left[c(j+k) 2^{-2 j_{w}} w^{-2 / 3} \bar{v} \bar{a}+c_{3} 2^{j} w^{-2 / 3} \bar{v} \bar{a} e^{-\bar{c} 2^{k / 2} / \varepsilon^{2}}\right. \\
& -\frac{a}{\bar{c} \varepsilon^{2} w} 2^{-\eta k}\left(\frac{\bar{v} \bar{a}+R}{c j^{2} k^{5}}\right)-\left(\frac{\bar{v} \bar{a}+R}{c j^{2} k^{5}}\right)^{2} \\
& \cdot\left(c_{1} c_{3} \varepsilon^{2} 2^{j} w^{-2 / 3} \bar{v} \bar{a} v w\right)^{-1} \exp \left[-c_{0}(\log N(a, w))^{2}\right]
\end{aligned}
$$

The third term in the exponential dominates the first, because $w^{1 / 3} \leqq c a^{1 / 2}$ $\leqq c a 2^{-2 \eta k}$; all factors $j$ or $k$ can be compensated with $2^{-2 j}$ or $2^{-\eta k}$. Similarly the third term dominates the second; we use also $j^{2} 2^{j} \leqq e^{\bar{c} 2^{k / 2} / \varepsilon^{2}}$. Finally, as in (4.37) we can bound

$$
j^{4} 2^{j} w^{-2 / 3} \bar{v} \bar{a} v w \leqq N(\bar{a}, \bar{w})^{4} 2^{N(\bar{a}, \bar{w})-3 k} w^{1 / 3} v \bar{v} \bar{a} \leqq c 2^{-3 k} \bar{v} \bar{w} w^{1 / 3} v \leqq c \bar{v} \bar{w}
$$

in the last term. (We use $v \leqq 2^{3 k} / w \leqq 2^{2 k}$.) This yields the following estimate:

$$
(4.39) \leqq c_{1} \exp \left[-\frac{(\bar{v} \bar{a}+R)^{2}}{c \varepsilon^{2} \bar{v} \bar{w}} k^{-10} \exp \left[-c_{0}(\log N(a, w))^{2}\right] .\right.
$$

If $j \geqq 2^{k / 2}$, then we use (4.21) only, yielding the bound (4.39) with only the first and last terms in the exponential. The last term dominates the first because its power of $2^{-j}$ is lower; its extra factors $w^{1 / 3} v^{-1}$ are bounded below by $j^{-4}$. We still have the bound (4.40), as it was derived using the last term in the exponential only. 
Next we consider the last term in (4.29), and let us suppose $N(\bar{a}, \bar{w})<2^{k / 2}$. It can be bounded as in (4.39):

$$
\begin{aligned}
\operatorname{Pr}\left(\exists \gamma: \Delta_{N}>\frac{\bar{v} \bar{a}+R}{c k^{5}}\right) \leqq & c_{1} \exp \left[c(j+k) 2^{-2 j} w^{-2 / 3} \bar{v} \bar{a}+c 2^{-3 k} \bar{v} \bar{w} e^{-\bar{c} 2^{k / 2} / \varepsilon^{2}}\right. \\
& -\frac{a}{\bar{c} \varepsilon^{2} w} 2^{-\eta k}\left(\frac{\bar{v} \bar{a}+R}{c k^{5}}\right)-\left(\frac{\bar{v} \bar{a}+R}{c k^{5}}\right)^{2}\left(c_{1} c \varepsilon^{2} 2^{-3 k} \bar{v} \bar{w} v w\right)^{-1} \\
& \left.\cdot \exp \left[-c_{0}(\log N(a, w))^{2}\right]\right]
\end{aligned}
$$

Here $j=\max \{0, N(a, w)-3 k\}$. The third term in the exponential dominates the first, as before. It dominates the second, because $\bar{w} / \bar{a} \leqq c 2^{3 N(\bar{a}, \bar{w}) / 2} \leqq c \exp \left(c 2^{k / 2}\right)$. Thus from the last term we obtain an estimate as in the right-hand side of (4.40). We use $v w \leqq 2^{3 k}$.

Finally we suppose $N(\bar{a}, \bar{w}) \geqq 2^{k / 2}$, and take only the first and last terms in the exponential in (4.41). Energy beats entropy because

$$
\begin{aligned}
(j+k) 2^{-2 j} w^{-2 / 3} & \leqq c N(\bar{a}, \bar{w}) 2^{-2 N(a, w)} 2^{6 k} \\
& \leqq c N(\bar{a}, \bar{w}) 2^{-N(a, w) / 2}(\bar{a} / \bar{w}) 2^{6 k} \leqq c k^{-10}(\bar{a} / \bar{w}),
\end{aligned}
$$

and we again obtain (4.40) as an upper bound.

We have proven the following estimate on (4.29):

$$
\begin{aligned}
\operatorname{Pr}\left(c_{\bar{k}, \bar{a}, \bar{w}, \bar{v}, \bar{x}}(h)<-R\right) \leqq & \exp \left[-\frac{(\bar{v} \bar{a}+R)^{2}}{c \varepsilon^{2} \bar{v} \bar{w}}\right]+\sum_{k, w, a, v, j} 2 c_{1} \\
& \cdot \exp \left[-\frac{(\bar{v} \bar{a}+R)^{2}}{c \varepsilon^{2} \bar{v} \bar{w}} k^{-10} \exp \left[-c_{0}(\log N(a, w))^{2}\right]\right] \\
\leqq & c \bar{k}^{5} \exp \left[-\frac{(\bar{v} \bar{a}+R)^{2}}{c \varepsilon^{2} \bar{v} \bar{w}} N(\bar{a}, \bar{w})^{-10}\right. \\
& \left.\cdot \exp \left[-c_{0}(\log 2 N(\bar{a}, \bar{w}) / 3)^{2}\right]\right] .
\end{aligned}
$$

Here we have used Lemma 4.6 and the bounds $w, a, v \leqq c 2^{3 k}, j \leqq N(a, w)$, $k \leqq c N(a, w)$. This last bound follows from $2^{k} \leqq w$ and the restriction $w<2^{3\left(N(\gamma)-N_{0}\right)}$ in (4.26). Putting $L=\log N(\bar{a}, \bar{w}), \log \frac{2}{3}=-\delta$, we have

$$
\begin{aligned}
& 2 c N(\bar{a}, \bar{w})^{10} \exp \left[c_{0}(\log 2 N(\bar{a}, \bar{w}) / 3)^{2}\right] \\
& \quad \leqq \exp \left[c_{0} L^{2}-2 c_{0} \delta L+c_{0} \delta^{2}+10 L+\log 2 c\right] \leqq \exp \left(c_{0} L^{2}\right),
\end{aligned}
$$

for $c_{0}$ a sufficiently large constant. (Note that $L>\delta$.) Hence (4.42) is bounded by

$$
c \bar{k}^{5} \exp \left[-\frac{2(\bar{v} \bar{a}+R)^{2}}{\varepsilon^{2} \bar{v} \bar{w}} \exp \left[-c_{0}(\log N(\bar{a}, \bar{w}))^{2}\right]\right],
$$

and the bound (4.1) easily follows. This completes the induction step in the proof of Proposition 4.1. 


\section{Entropy Estimates}

This section is devoted to proving Propositions 4.7 and 4.8. We begin with a lemma estimating the areas of our coarse-grained contours.

Lemma 5.1. For any external contour $\gamma$,

$$
\begin{gathered}
\left|\partial W_{j}(\gamma)\right| \leqq c 2^{-2 j}|\gamma|, \\
\left|\partial W_{k, w, j}(\gamma)\right| \leqq c 2^{-2 j} w^{-2 / 3}|\gamma| .
\end{gathered}
$$

Here $W_{j}(\gamma), W_{k, w, j}(\gamma)$ are regarded as subsets of $2^{j} \mathbb{Z}^{3}, 2^{j+k} \mathbb{Z}^{3}$, respectively.

Proof. The bound (5.1) was proven in [14]. It is essentially a special case $\left(w \approx 2^{3 k}\right)$ of the estimate

$$
\left|\partial V_{k, w}(\gamma)\right| \leqq c w^{-2 / 3}|\gamma|,
$$

which we now prove. If $\left(x, x^{\prime}\right) \in \partial V_{k, w}(\gamma)$, then $\left|B_{k, x}\right| V(\gamma)|<w,| B_{k, x^{\prime}}|V(\gamma)| \geqq w$, or vice versa. This implies that $U=B_{k, x} \cup B_{k, x^{\prime}}$ has the property that $w \leqq|U \backslash V(\gamma)|$ $<w+\frac{1}{8} 2^{3(k+3)}$. Putting $R=U \backslash V(\gamma)$, we have that $|\partial R| \geqq c w^{2 / 3}$. We claim that $|\gamma \cap \partial R| \geqq c w^{2 / 3}$ also. If we translate plaquettes of $\partial R \cap \gamma$ parallel to themselves to $\partial U$, we find that a nontrivial fraction of plaquettes of $\partial R \cap \partial U$ are covered. Otherwise we would have $|U \backslash V(\gamma)|$ approaching $|U|$, violating the inequality above. This implies that $|\partial R \cap \gamma| \geqq c|\partial R \cap \partial U|$, which yields the claim.

Only a few sets $U$ share portions of $\gamma$. This implies (5.3). To obtain (5.2), recall that $W_{k, w, j}(\gamma)$ is obtained from $V_{k, w}(\gamma)$ by the standard coarse-graining procedure, rescaled to the $2^{k}$-lattice. Hence we can combine (5.1) and (5.3) to obtain

$$
\left|\partial W_{k, w, j}(\gamma)\right| \leqq c 2^{-2 j}\left|\partial V_{k, w}(\gamma)\right| \leqq c 2^{-2 j} w^{-2 / 3}|\gamma| .
$$

The proof is completed by summing over $\gamma \in \bar{\gamma}$.

Proof of Proposition 4.7. We prove only (4.32), since (4.31) is similar (see [14]). Suppose that $y \in V_{k, w, j}(\bar{\gamma}) \backslash V_{k, w, j-1}(\bar{\gamma}), y \in 2^{k+j} \mathbb{Z}^{3}$. Then the cube $c_{k, j}(y)$ contains a pair of nearest neighbor cubes $c_{k, j-1}(x), c_{k, j-1}\left(x^{\prime}\right)$ with

$$
\frac{1}{2} 2^{3(j-1)}<\left|V_{k, w}(\gamma) \cap\left(c_{k, j-1}(x) \cup c_{k, j-1}\left(x^{\prime}\right)\right)\right| \leqq \frac{3}{2} 2^{3(j-1)},
$$

for some $\gamma \in \bar{\gamma}$.

As above, this implies that a section of $\partial V_{k, w}(\gamma)$ of size $c 2^{2 j}$ lies in these cubes. As in (5.3), each bond in $\partial V_{k, w}(\gamma)$ yields a section of $\gamma$ of size $w^{2 / 3}$. Since $\left|V_{k, w, j}(\gamma) \cap c_{k, j}(y)\right|=2^{3 j}$, we have

$$
\left|V_{k, w, j}(\gamma) \backslash V_{k, w, j-1}(\gamma)\right| \leqq c 2^{3 j} 2^{-2 j} w^{-2 / 3}|\gamma|,
$$

and the second half of (4.32) follows by summing over $\gamma \in \bar{\gamma}$. The first half is proven similarly.

Proof of Proposition 4.8. We again concentrate on the estimates for regions $V_{k, w, j}(\bar{\gamma})$, the estimates for $V_{j}(\bar{\gamma})$ being simpler. We show that the number of possible $V_{k, w, j}(\gamma)$ 's, as $\gamma$ varies over external contours belonging to $B_{k, x}$ with $|\gamma| \in[\bar{a}, 2 \bar{a})$, $|V(\gamma)| \in[\bar{w}, 2 \bar{w})$, is less than $\exp \left(c(j+k) 2^{-2 j} w^{-2 / 3} \bar{a}\right)$. This implies the bound in the proposition, because $V_{k, w, j}(\bar{\gamma})$ is a disjoint union of less than $2 \bar{v} V_{k, w, j}(\gamma)$ 's as above. 
A basic element of this proof is the following bound. Let $n(P, D)$ be the number of possible collections of contours built from $P$ plaquettes and such that there exists a walk $\left\{x_{i}\right\}_{1 \leqq i \leqq P}$ of length $D=\sum_{i=1}^{P} 2\left|x_{i}-x_{i-1}\right|$ connecting the centers of the plaquettes to a fixed point $x_{0}$. Then

$$
n(P, D) \leqq(c D / P)^{c P} .
$$

To prove this, consider the number of $P$-step walks of total length $D$. We have to partition $D$ into $D=\sum_{i=1}^{P} d_{i}$ with $d_{i} \in \mathbb{Z}, d_{i} \geqq 1$. The number of such partitions is $\left(\begin{array}{c}D-1 \\ P\end{array}\right) \leqq(c D / P)^{P}$. Given $\left\{d_{i}\right\}$, the number of walks is bounded by $\prod_{i=1}^{P}\left(c d_{i}\right)^{3}$ in three dimensions. Constraining the sum of the $d_{i}$ 's to be $D$, the product is maximized when the factors are equal. Hence it is bounded by $(c D / P)^{3 P}$. This yields (5.4).

We now estimate the number of possible $W_{k, w, j}(\gamma)$ 's, or equivalently, the number of possible collections of contours in $2^{k+j} \mathbb{Z}^{3}$ that could be $\partial W_{k, w, j}(\gamma)$. Recall our construction of external contours. Starting with an outer contour $\Gamma_{0} \rightarrow B_{\bar{k}, \bar{x}}$, we added outer contours $\gamma_{\alpha}$ in $\tilde{V}\left(\Gamma_{0}\right)$, either because $\gamma_{\alpha}$ is connected to a $\gamma_{\beta}$ with dist $\left(B\left(\gamma_{\beta}\right), \Gamma_{0}\right) \leqq 2^{k\left(\gamma_{\alpha}\right)+3}$ through a chain of pairs $\left(\gamma^{\prime}, \gamma^{\prime \prime}\right)$ with $\operatorname{dist}\left(B\left(\gamma^{\prime}\right), B\left(\gamma^{\prime \prime}\right)\right)$ $\leqq 2^{\min \left\{k\left(\gamma^{\prime}\right), k\left(\gamma^{\prime}\right)\right\}+3}$, or because $\left|\gamma_{\alpha}\right| \geqq 2^{2\left(N\left(\Gamma_{0}\right)-N_{0}\right)}$. This produced $\Gamma_{1}$, and if $N\left(\Gamma_{1}\right)$ $<N\left(\Gamma_{0}\right)$, then we continued with $\Gamma_{2}, \Gamma_{3}$, etc.

As a preliminary step, we choose one plaquette in $2^{k+j} \mathbb{Z}^{3}$ which is to be within a distance $2^{k+j}$ of $\Gamma_{0}$. There are less than $c 2^{3 \bar{k}} \leqq c \bar{a}^{3}$ possibilities. Next we choose the integers $N\left(\Gamma_{l}\right), l=0,1,2, \ldots$. These are a decreasing sequence, with $N\left(\Gamma_{0}\right) \leqq c k$ $\leqq c \log \bar{a}$. We also choose those $l$ such that $\Gamma_{l+1} \backslash \Gamma_{l}$ is to lie within a distance $2^{k+j}$ of $\partial W_{k, w, j}(\gamma)$. Denote these integers $l_{0}<l_{1}<\ldots$. There are at most $2^{c \log \bar{a}}$ choices of $\left\{N\left(\Gamma_{l}\right)\right\}$, and similarly for the choices of $\left\{l_{\mu}\right\}$. Hence all these initial choices can be subsumed in a factor $\bar{a}^{c} \leqq \exp \left(c(j+k) 2^{-2 j} w^{-2 / 3} \bar{a}\right)$. We are using the fact that

$$
2^{2 j} w^{2 / 3} \leqq 2^{2 j+2 k} \leqq 2^{2 N(\bar{a}, \bar{w})} \leqq c(\bar{w} / \bar{a})^{4 / 3} \leqq c \bar{a}^{2 / 3} \leqq c \bar{a} .
$$

We will connect the plaquettes of $\partial W_{k, w, j}(\gamma)$ to the chosen plaquette through a tree graph of steps connecting centers of plaquettes. First we describe the trunk $\left\{t_{i}\right\}, i=1,2, \ldots$, which is a walk of some of the plaquettes, starting at $t_{0}$, the center of the chosen plaquette. Then we describe the branches, which are walks starting at the $t_{i}$ 's.

The first few steps connect $t_{0}$ to some plaquettes within $2^{k+j}$ of $\Gamma_{l_{0}+1} \backslash \Gamma_{l_{0}}$. We break $\Gamma_{l_{0}+1} \backslash \Gamma_{l_{0}}$ into components as follows: Contours $\gamma_{\alpha}, \gamma_{\beta}$ in $\Gamma_{l_{0}+1} \backslash \Gamma_{l_{0}}$ are connected if

$$
\operatorname{dist}\left(B\left(\gamma_{\alpha}\right), B\left(\gamma_{\beta}\right)\right) \leqq 2^{\min \left\{k\left(\gamma_{\alpha}\right), k\left(\gamma_{\beta}\right)\right\}+3} .
$$

When $l_{0}=0$, we exclude the components that would be connected to $\Gamma_{0}$. For each remaining component, we choose one plaquette within a distance $2^{k+j}$, if this is possible. By construction, each component should have area at least $2^{2\left(N\left(\Gamma_{l_{0}}\right)-N_{0}\right)}$. Hence there are no more than

$$
c\left|\Gamma_{l_{0}+1} \backslash \Gamma_{l_{0}}\right|^{-2 N\left(\Gamma_{l_{0}}\right)} \leqq c\left|\Gamma_{l_{0}+1}\right| 2^{-2 N\left(\Gamma_{l_{0}}\right)}
$$


components. These are supposed to lie in $V\left(\Gamma_{l_{0}}\right)$, which is connected, hence the plaquettes can be walked with a distance less than $c\left|V\left(\Gamma_{l_{0}}\right)\right|$. By (5.4), rescaled to the lattice $2^{k+j} \mathbb{Z}^{3}$, the number of such walks is less than

$$
\begin{aligned}
\left(\frac{c\left|V\left(\Gamma_{l_{0}}\right)\right| 2^{-k-j}}{\left|\Gamma_{l_{0}+1}\right| 2^{-2 N\left(\Gamma_{l_{0}}\right)}}\right)^{c\left|\Gamma_{l_{0}+1}\right| 2^{-2 N\left(\Gamma_{l_{0}}\right)}} & \leqq\left(\frac{c V\left(\Gamma_{l_{0}}\right)}{\left|\Gamma_{l_{0}}\right|} 2^{2 N\left(\Gamma_{l_{0}}\right)}\right)^{c \bar{a} 2-2 N\left(\Gamma_{l_{0}}\right)} \\
& \leqq \exp \left(c N\left(\Gamma_{l_{0}}\right) 2^{-2 N\left(\Gamma_{l_{0}}\right)} \bar{a}\right)
\end{aligned}
$$

We continue choosing the next sections of the trunk $\left\{t_{i}\right\}$. We choose plaquettes near components of each $\Gamma_{l_{\mu}+1} \backslash \Gamma_{l_{\mu}}, \mu=1,2, \ldots$. The number of choices for the $\mu^{\text {th }}$ section is bounded as above by $\exp \left(c N\left(\Gamma_{l_{\mu}}\right) 2^{-2 N\left(\Gamma_{l_{\mu}}\right)} \bar{a}\right)$. Hence the total number of possible trunks is bounded by

$$
\exp \left(\sum_{\mu} c N\left(\Gamma_{l_{\mu}}\right) 2^{-2 N\left(\Gamma_{l_{\mu}}\right)} \bar{a}\right) \leqq \exp \left(c N(\gamma) 2^{-2 N(\gamma)} \bar{a}\right)
$$

since $N\left(\Gamma_{l_{\mu}+1}\right) \leqq N\left(\Gamma_{l_{\mu}}\right)-1, N\left(\Gamma_{l}\right) \geqq N(\gamma)$. Finally, we note that by $(5.5), 2 N(\gamma)+c$ $\geqq 2 j+\frac{2}{3} \log _{2} w$, so that $(5.7)$ is bounded by $\exp \left(c(j+k) 2^{-2 j} w^{-2 / 3} \bar{a}\right)$.

Having estimated the entropy for positions of the large contours in $V\left(\Gamma_{0}\right)$, we can proceed to choose the rest of $\partial W_{k, w}, j(\gamma)$. The trunk has been constructed so that at most a distance $c|\gamma|$ is required to connect the rest of $\partial W_{k, w, j}(\gamma)$ to it. This is because every plaquette in $\partial W_{k, w, j}(\gamma)$ must lie within $2^{k+j}$ of a portion of $\gamma$ that is connected as above and close to some $t_{i}$. Each such portion of $\gamma$ can be walked in a distance of the order of its area - large steps are needed only to reach comparably large contours. Hence the sum of the lengths of the required branch walks is less than $c|\gamma|$.

The total number of plaquettes in $\partial W_{k, w, j}(\gamma)$ is less than $P=c 2^{-2 j} w^{-2 / 3}|\gamma|$. Certainly this is also a bound on the number of steps in the trunk. Hence a factor $\exp \left(c 2^{-2 j} w^{-2 / 3}|\gamma|\right)$ allows us to choose which steps in the trunk will acquire branches. We need to partition the plaquettes for attachment into subsets which will form the branches. There are at most $2^{P}$ possibilities, again bounded by $\exp \left(c 2^{-2 j} w^{-2 / 3}|\gamma|\right)$. We are now in a situation considered in the single walk case, having to choose at most $P$ steps of total length less than $c|\gamma|$, or $c 2^{-k-j}|\gamma|$ in $2^{k+j} \mathbb{Z}^{3}$. Hence the number of possible sets of branches, with starting points and numbers of steps specified as above, is less than

$$
\left(\frac{c D}{P}\right)^{P} \leqq\left(\frac{c 2^{-k-j}|\gamma|}{2^{-2 j} w^{-2 / 3}|\gamma|}\right)^{c 2^{-2 j_{w}-2 / 3}|\gamma|} \leqq \exp \left(c(j+k) 2^{-2 j} w^{-2 / 3} \bar{a}\right)
$$

Gathering together all combinatoric factors, we obtain an estimate $\exp \left(c(j+k) 2^{-2 j} w^{-2 / 3} \bar{a}\right)$ on the number of possible $W_{k, w, j}(\gamma)$ 's. This completes the proof.

\section{Long-Range Order in the Random-Field Ising Model}

We are now in a position to prove Theorems 1.1 and 1.2. Let us avoid the set of measure zero on which the ground state configuration in $\Lambda^{+}$is non-unique. We have the following formula for the ground state configuration $\sigma^{\min }\left(\Lambda^{+}\right)$:

$$
\sigma_{i}^{\min }\left(\Lambda^{+}\right)=\left\{\begin{aligned}
-1, & \text { if } i \in S(\gamma) \text { for some favored }(+) \text { external contour } \gamma \\
1, & \text { otherwise }
\end{aligned}\right.
$$


Here $S(\gamma)=\left\{i \in V(\gamma): \sigma_{i}^{\min }\left(V(\gamma)^{+}\right)=-1\right\}$. This formula holds because as in the proof of Lemma $2.1, S(\gamma) \subseteq S\left(\gamma^{\prime}\right)$, where $\gamma^{\prime}$ is the maximal $\left(\cong \Lambda^{+}\right)$external contour with $V(\gamma) \subseteq V\left(\gamma^{\prime}\right)$. By Lemma 2.2, as in the proof of Proposition 3.2, we have $\sigma^{\min }\left(V\left(\gamma^{\prime}\right)^{+}\right)=\sigma^{\min }\left(\Lambda^{+}\right)\left\lceil V\left(\gamma^{\prime}\right)\right.$, which yields (6.1).

Proof of Theorem 1.1. From (6.1) and the fact that $r_{y} \leqq 0$ [strict inequality only if $\gamma$ is favored $(+)]$, we have an estimate

$$
\operatorname{Pr}\left(\sigma_{i}^{\min }\left(\Lambda^{+}\right)=-1\right) \leqq \operatorname{Pr}\left(\sum_{\gamma: i \in V(\gamma)} r_{\gamma}<0\right) \leqq \operatorname{Pr}\left(\sum_{k, x: i \in B_{k, x}} \sum_{a, w, v} r_{k, a, w, v, x}<0\right) .
$$

We apply (4.2) and our main estimate, Proposition 4.1, to bound this by

$$
\begin{aligned}
\sum_{k, a, w, v, x} & \exp \left\{-\frac{v a^{2}}{\varepsilon^{2} w} \exp \left[-c_{0}(\log N(a, w))^{2}\right]\right\} \\
& \leqq \sum_{k=0}^{\infty} c k^{3} \exp \left(-c 2^{k(1-\eta)} / \varepsilon^{2}\right) \leqq \exp \left(-C / \varepsilon^{2}\right) .
\end{aligned}
$$

This yields (1.3) and the theorem.

Next we prove a strong result about the approach to the infinite volume limit.

Theorem 6.1. The limit

$$
\lim _{n \rightarrow \infty} \sigma_{i}^{\min }\left(\Lambda^{+}\right) \equiv \sigma_{i}^{\min }
$$

exists for each $i \in \mathbb{Z}^{3}$, with probability 1 . Furthermore, there exists a constant $C>0$ such that for any $n$,

$$
\operatorname{Pr}\left(\exists m \geqq 2 n: \sigma_{i}^{\min }\left(\Lambda_{m}^{+}\right) \neq \sigma_{i}^{\min } \text { for any } i \in \Lambda_{n}\right) \leqq \exp \left(-C n^{1-\eta} / \varepsilon^{2}\right) .
$$

Consequently, for any $\mathscr{A} \subset \mathbb{Z}^{3}$ with $\max _{i \in \mathscr{A}}|i| \leqq n / 2$,

$$
\left|\prod_{i \in \mathscr{A}} \sigma_{i}^{\min }\left(\Lambda_{n}^{+}\right)-\prod_{i \in \mathscr{A}} \sigma_{i}^{\mathrm{min}}\right| \leqq \exp \left(-C n^{1-\eta} / \varepsilon^{2}\right) .
$$

Proof. Let us define provisionally $\sigma_{i}^{\mathrm{min}}$ by the right-hand side of (6.1), where we put $\Lambda=\mathbb{Z}^{3}$ in the definition of favored $(+)$. [Thus $\gamma$ is favored $(+)$ if it is favored in $B(\gamma)^{+}$instead of $(B(\gamma) \cap A)^{+}$.] Then if we verify (6.4), we obtain (6.3) automatically: To see that the limit exists with probability 1 we need only observe that the following statement is true. For any $\delta>0$ there exists an $n$ such that with probability $1-\delta, \sigma_{i}^{\min }\left(\Lambda_{m}^{+}\right)=\sigma_{i}^{\min }$ for all $m \geqq 2 n$.

We now prove (6.4). By (6.1) and the analogous statement for $\sigma^{\min }, \sigma_{i}^{\min }\left(\Lambda_{m}\right)$ can differ from $\sigma_{i}^{\text {min }}$ for some $i \in \Lambda_{n}$ only if there is an external contour $\gamma$ with $V(\gamma) \cap \Lambda_{n} \neq \emptyset$ which is favored (+) in $\Lambda_{m}$ but not in $\mathbb{Z}^{3}$, or vice versa. If $V(\gamma) \cap \Lambda_{n} \neq \emptyset$, then $B(\gamma) \backslash \Lambda_{m} \neq \emptyset$ only if $2^{k(\gamma)} \geqq(m-n) / 8$. Only such external contours can feel the difference between $\Lambda_{m}$ and $\mathbb{Z}^{3}$. Hence

$$
\begin{aligned}
\operatorname{Pr} & \left(\exists m \geqq 2 n: \sigma_{i}^{\min }\left(\Lambda_{m}^{+}\right) \neq \sigma_{i}^{\min } \text { for any } i \in \Lambda_{n}\right) \\
\leqq & \sum_{m \geqq 2 n} \sum_{k \geqq \log _{2}(m-n) / 8} \sum_{x: B_{k, x} \cap \Lambda_{n} \neq \phi} \sum_{a, w, v} \\
& \cdot\left[\operatorname{Pr}\left(r_{k, a, w, v, x}^{\left(\Lambda_{m}\right)}<0\right)+\operatorname{Pr}\left(r_{k, a, w, v, x}^{\left(\mathbb{Z}^{3}\right)}<0\right)\right] .
\end{aligned}
$$


Here the superscripts on the $r$ 's indicate the volume used in defining that random variable. Note that $r_{k, a, w, v, x}^{(\Lambda)}$ is independent of $\Lambda$ for $B_{k, x} \subset \Lambda$. Proposition 4.1 yields the following bound on (6.6):

$$
\begin{aligned}
& \sum_{m \geqq 2 n} \sum_{k \geqq \log _{2}(m-n) / 8} c n^{3} k^{3} \exp \left(-c 2^{k(1-\eta)} / \varepsilon^{2}\right) \\
& \quad \leqq \sum_{m \geqq 2 n} c n^{3} \exp \left(-c m^{1-\eta} / \varepsilon^{2}\right) \leqq \exp \left(-c n^{1-\eta} / \varepsilon^{2}\right) .
\end{aligned}
$$

This yields (6.4), completing the proof of the theorem.

Proof of Theorem 1.2. Defining $\tau_{i}=\left(1-\sigma_{i}^{\mathrm{min}}\right) / 2$, we easily obtain (1.9) from the estimate

$$
\left|\overline{\tau_{i} \tau_{j}}-\bar{\tau}_{i} \bar{\tau}_{j}\right| \leqq \exp \left(-c|i-j|^{1-\eta} / \varepsilon^{2}\right)
$$

We have that

$$
\tau_{i}= \begin{cases}1, & \text { if } i \in S(\gamma) \text { with } \gamma \text { favored }(+), \\ 0, & \text { otherwise. }\end{cases}
$$

Similarly $\tau_{i} \tau_{j}=1$ precisely when both sites lie in some $S(\gamma), \gamma$ favored $(+)-$ not necessarily the same $\gamma$. Let $E_{i}$ denote the event " $i \in S(\gamma), \gamma$ favored $(+)$ ", and let $e_{i}$ denote the event " $i \in S(\gamma), \gamma$ favored $(+), k(\gamma) \leqq \log _{2}|i-j| / 16$ ". Define similarly $E_{j}, e_{j}$. We have

$$
\begin{aligned}
\overline{\tau_{i} \tau_{j}} & =\operatorname{Pr}\left(E_{i} \text { and } E_{j}\right) \\
& =\operatorname{Pr}\left(e_{i} \text { and } e_{j}\right)+\operatorname{Pr}\left(E_{i} \text { and } E_{j} \text { but not } e_{i} \text { or } e_{j}\right) \\
& =\operatorname{Pr}\left(e_{i}\right) \operatorname{Pr}\left(e_{j}\right)+\operatorname{Pr}\left(E_{i} \text { and } E_{j} \text { but not } e_{i} \text { or } e_{j}\right),
\end{aligned}
$$

where in the last step we have used independence of $e_{i}, e_{j}$, which depend only on $h_{l}$ for $|i-l|<|i-j| / 2$, respectively $|j-l|<|i-j| / 2$. In a similar fashion we have

$$
\bar{\tau}_{i}=\operatorname{Pr}\left(E_{i}\right)=\operatorname{Pr}\left(e_{i}\right)+\operatorname{Pr}\left(E_{i} \text { but not } e_{i}\right)
$$

and the analogous statement with $j$ replacing $i$. Hence

$$
\begin{aligned}
\left|\overline{\tau_{i} \tau_{j}}-\bar{\tau}_{i} \bar{\tau}_{j}\right|= & \mid \operatorname{Pr}\left(E_{i} \text { and } E_{j} \text { but not } e_{i} \text { or } e_{j}\right) \\
& -\operatorname{Pr}\left(e_{i}\right) \operatorname{Pr}\left(E_{j} \text { but not } e_{j}\right)-\operatorname{Pr}\left(e_{j}\right) \operatorname{Pr}\left(E_{i} \text { but not } e_{i}\right) \\
& +\operatorname{Pr}\left(E_{i} \text { but not } e_{i}\right) \operatorname{Pr}\left(E_{j} \text { but not } e_{j}\right) \mid \\
\leqq & \operatorname{Pr}\left(E_{j} \text { but not } e_{j}\right)+\operatorname{Pr}\left(E_{i} \text { but not } e_{i}\right) \\
\leqq & \sum_{k>\log _{2}|i-j| / 16} \sum_{x: i \in B_{k, x} \text { or } j \in B_{k, x}} \sum_{a, w, v} 2 \operatorname{Pr}\left(r_{k, a, w, v, x}<0\right) \\
\leqq & \sum_{k>\log _{2}|i-j| 1 / 16} c k^{3} \exp \left(-c 2^{k(1-\eta) / \varepsilon^{2}}\right) \\
\leqq & \exp \left(-c|i-j|^{1-\eta} / \varepsilon^{2}\right),
\end{aligned}
$$

which is (6.8).

Finally, it is worth describing what our expansion yields about the free energy. Using the subadditive ergodic theorem [17], it can be shown that the free energy per site exists and is independent of the magnetic field configuration with 
probability 1 (similarly for the magnetization per site). We can see this directly from our expansion, with precise estimates on the rate of approach following from the bounds of Sect. 4. Define the free energy per site as

$$
f_{\Lambda_{n}} \equiv\left|\Lambda_{n}\right|^{-1} \inf _{\sigma\left\lceil\Lambda_{n}\right.} H^{+}\left(\Lambda_{n}\right) \text {. }
$$

By (3.1), (3.6) we have

$$
\inf _{\sigma \mid \Lambda_{n}} H^{+}\left(\Lambda_{n}\right)=\sum_{\gamma: V(\Lambda) \cong \Lambda_{n}} r_{\gamma}-\sum_{i \in \Lambda_{n}} \frac{1}{2} h_{i} .
$$

Hence

$$
f_{\Lambda_{n}}=\left|\Lambda_{n}\right|^{-1} \sum_{k, a, w, v, x} r_{k, a, w, v, x}-\left|\Lambda_{n}\right|^{-1} \sum_{i \in \Lambda_{n}} \frac{1}{2} h_{i} .
$$

The second term converges to zero, with probability 1 , by the central limit theorem. For each $k, a, w, v$ there are $8^{3}$ sublattices of $2^{k} \mathbb{Z}^{3}$ on which the $r_{k, a, w, v, x}$ 's are independent random variables. They are identically distributed, except for $x$ with dist $\left(x, \partial \Lambda_{n}\right)<2^{k+2}$. But the distribution of these $r_{k, a, w, v, x}$ 's obey uniform bounds (by Proposition 4.1 and the inequalities $c_{k, a, w, v, x} \leqq r_{k, a, w, v, x} \leqq 0$ ). Hence the boundary terms approach zero. Applying again the central limit theorem, we have

$$
f_{\Lambda_{n}} \rightarrow f_{\infty}=\sum_{k, a, w, v} 2^{-3 k} \overline{r_{k, a, w, v, 0}},
$$

where $r_{k, a, w, v, 0}$ is calculated in any $\Lambda_{n}$ with $n>2^{k+2}$. This limit is of course independent of the magnetic field configuration. Proposition 4.1 easily yields

$$
0 \geqq \overline{r_{k, a, w, v, 0}} \geqq-\exp \left(-c 2^{k(1-\eta)} / \varepsilon^{2}\right)
$$

which implies that

$$
-\exp \left(-C / \varepsilon^{2}\right) \leqq f_{\infty} \leqq 0 .
$$

Acknowledgements. The author would like to thank T. Spencer and J. Fröhlich for their encouragement and collaboration in related work, and for numerous discussions which made this work possible.

\section{References}

1. Berretti, A.: Some properties of random Ising models. J. Stat. Phys. (to appear)

2. Imry, Y., Ma, S.: Random-field instability of the ordered state of continuous symmetry. Phys. Rev. Lett. 35, 1399-1401 (1975)

3. Imry, Y.: Random external fields. J. Stat. Phys. 34, 849-862 (1984)

Birgenau, R., Cowley, R., Shirane, G., Yoshizawa, H.: Phase transitions in diluted magnets: critical behavior, percolation, and random fields. J. Stat. Phys. 34, 817-847 (1984)

Grinstein, G.: On the lower critical dimension of the random field Ising model. J. Appl. Phys. 55, 2371-2376 (1984)

4. Parisi, G., Sourias, N.: Random magnetic fields, supersymmetry, and negative dimensions. Phys. Rev. Lett. 43, 744-745 (1979)

5. Parisi, G., Sourlas, N.: Supersymmetric field theories and stochastic differential equations. Nucl. Phys. B206, 321-332 (1982)

6. Cardy, J.: Nonperturbative effects in a scalar supersymmetric theory. Phys. Lett. 125B, 470-472 (1983) 
7. Klein, A., Perez, J.: Supersymmetry and dimensional reduction: a non-perturbative proof. Phys. Lett. 125B, 473-475 (1983)

8. Klein, A., Landau, L., Perez, J.: Supersymmetry and the Parisi-Sourlas dimensional reduction: a rigorous proof. Commun. Math. Phys. 94, 459-482 (1984)

9. Pytte, E., Imry, Y., Mukamel, D.: Lower critical dimension and the roughening transition of the random-field Ising model. Phys. Rev. Lett. 46, 1173-1177 (1981)

10. Mukhamel, D., Pytte, E.: Interface fluctuations and the Ising model in a random field. Phys. Rev. B25, 4779-4786 (1982)

11. Grinstein, G., Ma, S.: Roughening and lower critical dimension in the random-field Ising model. Phys. Rev. Lett. 49, 685-688 (1982)

12. Grinstein, G., Ma, S.: Surface tension, roughening, and lower critical dimension in the random-field Ising model. Phys. Rev. B28, 2588-2601 (1983)

13. Villain, J.: Commensurate-incommensurate transition with frozen impurities. J. Phys. 43, L551-L558 (1982)

14. Fisher, D., Fröhlich, J., Spencer, T.: The Ising model in a random magnetic field. J. Stat. Phys. 34, 863-870 (1984)

15. Fröhlich, J., Imbrie, J.: Improved perturbation expansion in disordered systems: Beating Griffiths singularities. Commun. Math. Phys. 96, 145-180 (1984)

16. Chalker, J.: On the lower critical dimensionality of the Ising model in a random field. J. Phys. C16, 6615-6622 (1983)

17. van Enter, A., van Hemmen, J.: The thermodynamic limit for long-range random systems. J. Stat. Phys. 32, 141-152 (1983)

18. Krey, U.: On the lower critical dimension of spin systems in random fields. J. Phys. C (to appear)

Communicated by A. Jaffe

Received July 26, 1984; in revised form October 22, 1984 